\title{
Rigorous derivation of a hyperbolic model for Taylor dispersion
}

\author{
Andro Mikelić ${ }^{* \dagger}$ \\ Université de Lyon, Lyon, F-69003, FRANCE ; \\ Université Lyon 1, Institut Camille Jordan, \\ UMR 5208, UFR Mathématiques, 43, Bd du 11 novembre 1918, \\ 69622 Villeurbanne Cedex, France

\section{C.J. van Duijn} \\ Eindhoven University of Technology, \\ Department of Mathematics and Computer Sciences, \\ P.O. Box 513, 5600 MB Eindhoven, The Netherlands
}

October 13, 2009

\begin{abstract}
In this paper we upscale the classical convection-diffusion equation in a narrow slit. We suppose that the transport parameters are such that we are in Taylor's regime i.e. we deal with dominant Peclet numbers. In contrast to the classical work of Taylor, we undertake a rigorous derivation of the upscaled hyperbolic dispersion equation. Hyperbolic effective models were proposed by several authors and our goal is to confirm rigorously the effective equations derived by Balakotaiah et al in recent years using a formal Liapounov - Schmidt reduction. Our analysis uses the Laplace transform in time and an anisotropic singular perturbation technique, the small characteristic parameter $\varepsilon$ being the ratio between the thickness and the longitudinal observation length. The Péclet number is written as $C \varepsilon^{-\alpha}$, with $\alpha<2$. Hyperbolic effective model corresponds to a high Péclet number close to the threshold value when Taylor's regime turns to turbulent mixing and we characterize it by supposing $4 / 3<\alpha<2$. We prove that the difference between the dimensionless physical concentration and the effective concentration, calculated using the hyperbolic upscaled model, divided by $\varepsilon^{2-\alpha}$ (the local Péclet number) converges strongly to zero in $L^{2}$-norm. For Péclet numbers considered in this paper, the hyperbolic dispersion equation turns out to give a better approximation than the classical parabolic Taylor model.
\end{abstract}

* Research of A.M. was partially supported by the GNR MOMAS CNRS-2439 (Modélisation Mathématique et Simulations numériques liées aux problèmes de gestion des déchets nucléaires) (PACEN/CNRS, ANDRA, BRGM, CEA, EDF, IRSN).

${ }^{\dagger}$ E-mail: Andro.Mikelic@univ-lyon1.fr 
Keywords Taylor's dispersion; Goursat problem, large Peclet number; singular perturbation; Laplace's transform; Danckwerts' boundary conditions. AMS classcode 35B25; 76F25; 44A10

\section{Introduction}

Aim of this paper is to present an upscaled hyperbolic model for Taylor dispersion.

Dispersion expresses the deviation of a solute concentration with respect to its mean behavior. It is induced by the motion of a fluid that transports the solute (molecular diffusion, convection and their interaction) or by chemical reactions.

Dispersion induced by a flow between two parallel plates (or through a tube) is classically modeled by an effective convection-diffusion equation of the type

$$
\frac{\partial c}{\partial t}+<v>\frac{\partial c}{\partial x}=\tilde{D}_{e f f} \frac{\partial^{2} c}{\partial x^{2}}
$$

Here $\langle v\rangle$ denotes the transversally averaged velocity and $\tilde{D}_{\text {eff }}$ the effective dispersion coefficient.

The latter depends on the transversal Péclet number $\mathbf{P e}_{T}=\frac{\langle v>H}{D_{m o l}}$, where $D_{m o l}$ is the molecular diffusivity and $H$ vertical distance (tube radius). In his pioneering paper [29], Taylor found for tracer flow in a narrow tube that $\tilde{D}_{\text {eff }}$ behaves as $D_{\text {mol }}\left(1+C \mathbf{P e}_{T}^{2}\right)$. Here $C$ is explicitly known, depending on the geometry. This expression for $\tilde{D}_{e f f}$ is believed to hold until $\mathbf{P e}_{T}$ reaches a threshold value. Crossing that value, turbulent mixing appears and the dependence on $\mathbf{P e}_{T}$ becomes either logarithmic, sublinear or linear (see [30] and chapter 9 from [17]).

In this work we study the dispersion between two parallel plates for Péclet numbers in the Taylor regime, i.e. below the threshold value but nevertheless close to it. This in fact is the situation discussed in the classical Taylor paper [29].

In that paper, he considered the transport of a solute by Poiseuille flow in the presence of transversal (molecular) diffusion. The effective Taylor equation was derived for the cross-section averaged solute concentration. Taylor derived and experimentally verified, that for a cylindrical tube with radius $H$ the effective equation reads

$$
\frac{\partial c}{\partial t}+<v>\frac{\partial c}{\partial x}-D_{m o l} \frac{\partial^{2} c}{\partial x^{2}}=-\frac{\partial J_{T}}{\partial x},
$$

where the additional dispersion flux $J_{T}$ is given by

$$
J_{T}=-D_{e f f} \frac{\partial c}{\partial x}=-\frac{H^{2}<v>^{2}}{48 D_{m o l}} \frac{\partial c}{\partial x} .
$$


This expression was formally justified by Aris in [3], using the method of spatial moments.

In this paper we present an alternative for the upscaled model in the Taylor regime which is of the form

$$
\frac{\partial c}{\partial t}+<v>\frac{\partial c}{\partial x}+<v>\frac{H^{2}}{48 D_{m o l}} \frac{\partial^{2} c}{\partial x \partial t}=0 .
$$

For reasons of simplicity we will derive the analogue of (4) in the setting of a Poiseuille flow between two parallel plates. Also the error estimates are done within this simplified setting.

There is a large number of papers related to Taylor dispersion, but only a few are concerned with a rigorous mathematical justification of the effective model. To guide the reader through the literature we present below a brief summary. We start by mentioning the center manifold approach of Mercer and Roberts [22] and the related paper [26] by Rosencrans. This approach allows one to calculate approximations at any order for the original Taylor model. Even though no error estimate was obtained, this approach gives a plausible argument for the validity of the effective model. A rigorous justification of the effective dispersion model including chemical reactions on the wall of the slit, was undertaken in [23] by Mikelić et al. An anisotropic singular perturbation technique was used and the results of this paper cover the classical Taylor case (1) and (2), as well.

Dispersion for reactive flows in tubes was studied by Paine et al. in [25]. They noted that the equation for the difference between the actual physical and averaged concentrations is not closed, since it contains a dispersive source term. They used the "single-point" closure schemes of turbulence modeling by Launder [19] to obtain a closed model for the averaged concentration. We note that their effective equations contain non-local terms depending on the solution. In fact the effective coefficients are not explicitly given.

The center manifold approach was applied to reactive flows by Balakotaiah and Chang in [4]. A number of effective models for different Damköhler numbers were obtained, where the Damköhler number is the ratio of the characteristic reaction time and the characteristic transversal diffusion time. In [15] by van Duijn et al, the case of general chemical reactions was considered from the point of view of formal expansions with respect to the local Péclet number, being the ratio between the characteristic longitudinal transport time and transversal diffusion time. Effective dispersion equations were obtained and the results were justified by numerical simulations, where the direct simulation of the physical multidimensional problem was compared to the solution of the effective dispersion equations. An excellent agreement was found. The expansion and numerical simulation results were justified at the level of mathematical rigor in the papers [12], [13], [23] and [24]. The analysis uses an anisotropic singular perturbation method to obtain error estimates for the approximations. At high Péclet numbers the presence of the inlet boundaries required construction of boundary layers and this led to severe technical complications. 
Characteristic to all these models is that they give rise to parabolic transport equations for the effective solute concentration.

Consequently, this leads to some non-physical properties:

i) In the starting equation the longitudinal diffusion is frequently neglected. Nevertheless, the dispersion equations have effective dispersion in the longitudinal direction. Consequently, they predict infinite propagation speed of perturbations, which is (of course) not observed in experiments.

ii) For purely convective flows solute particles follows streamlines. Hence when flow reversal occurs the particules return to their original position. Obviously, this is not true anymore in the presence of transversal diffusion, since then particle move randomly in the transverse direction between streamlines. One speaks of partial reversibility if directly after flow reversal a variance decrease is observed. On physical grounds one expects to have at least partial reversibility.

These reasons motivated Scheidegger [28] to propose already in 1958 the onedimensional telegraph equation

$$
\frac{\partial^{2} c}{\partial t^{2}}+\frac{1}{\tau} \frac{\partial c}{\partial t}=\sigma_{v}^{2} \frac{\partial^{2} c}{\partial x^{2}}
$$

as model for dispersion in porous media. Here $\sigma_{v}$ is the velocity variance and $\tau$ a relaxation parameter.

For dispersion in a tube, Camacho developed in [8]-[10] upscaled hyperbolic models. He used concepts from irreversible thermodynamics and he averaged the terms of the Fourier expansion of the solution in the original equation over the cross-section of the flow. In this way he arrived at the following non-Fickian relaxation equation for the Taylor flux $J_{T}$ :

$$
\begin{gathered}
\frac{\partial J_{T}}{\partial t}+\frac{J_{T}}{\tau}+(1+\beta)<v>\frac{\partial J_{T}}{\partial x} \\
-D_{m o l} \frac{\partial^{2} J_{T}}{\partial x^{2}}=-\sigma_{v}^{2} \frac{\partial^{2} c}{\partial x^{2}} .
\end{gathered}
$$

Here $\tau$ is again relaxation parameter time and $\beta$ is a phenomenological coefficient. After some simplifications and manipulations, (6) leads to a fourth order equation for the averaged concentration:

$$
\begin{gathered}
\frac{\partial c}{\partial t}+<v>\frac{\partial c}{\partial x}-D_{m o l} \frac{\partial^{2} c}{\partial x^{2}}+\tau_{e f f}\left\{\frac{\partial^{2} c}{\partial t^{2}}+\left(\left(<v>+\beta_{e f f}\right)<v>-\sigma_{v}^{2}\right) \frac{\partial^{2} c}{\partial x^{2}}\right. \\
\left.+\left(2<v>+\beta_{e f f}\right) \frac{\partial^{2} c}{\partial x \partial t}\right\} \\
=\tau_{e f f}\left\{2 D_{m o l} \frac{\partial^{3} c}{\partial x^{2} \partial t}+\left(2<v>+\beta_{e f f}\right) D_{m o l} \frac{\partial^{3} c}{\partial x^{3}}-D_{m o l}^{2} \frac{\partial^{4} c}{\partial x^{4}}\right\}
\end{gathered}
$$


Here $\beta_{\text {eff }}$ and $\tau_{\text {eff }}$ are effective parameters related to $\beta$ and $\tau$. Neglecting molecular diffusion reduces (7) to the telegraph equation

$$
\begin{gathered}
\tau_{e f f} \frac{\partial^{2} c}{\partial t^{2}}+\frac{\partial c}{\partial t}+<v>\frac{\partial c}{\partial x}+\tau_{e f f}\left(2<v>+\beta_{e f f}\right) \frac{\partial^{2} c}{\partial x \partial t}- \\
\tau_{e f f}\left(\sigma_{v}^{2}-\left(<v>+\beta_{e f f}\right)<v>\right) \frac{\partial^{2} c}{\partial x^{2}}=0
\end{gathered}
$$

The results of Camacho were later extended to layered media by Berentsen et al in [7].

An alternative approach, based on a two-term Bubnov-Galerkin representation, was introduced by Khon'kin in [18]. For dispersion in a tube his calculations lead to a hyperbolic equation of the form

$$
\frac{\partial^{2} c}{\partial t^{2}}+\frac{9}{4}<v>\frac{\partial^{2} c}{\partial x \partial t}+\frac{15}{16}<v>^{2} \frac{\partial^{2} c}{\partial x^{2}}+\frac{15 D_{m o l}}{H^{2}}\left(\frac{\partial c}{\partial t}+<v>\frac{\partial c}{\partial x}\right)=0
$$

Yet another approach, similar but more systematic, was developed by Balakotaiah et al. in [5], [6] and [11]. They use the Liapounov-Schmidt reduction together with a perturbation argument. In the framework of Taylor's paper they obtained the dispersion equation

$$
\frac{\partial c}{\partial t}+<v>\frac{\partial c}{\partial x}+<v>\frac{H^{2}}{48 D_{m o l}} \frac{\partial^{2} c}{\partial x \partial t}=D_{m o l} \frac{\partial^{2} c}{\partial x^{2}}
$$

Since $D_{m o l}$ is very small, the right hand side could be disregarded. In this approximation equation (10) reduces to our equation (4). In this hyperbolic limit, equation (10) does not suffer from default i) and has partial reversibility.

In this paper we address the rigorous mathematical justification of the formal results and observations made by Balakotaiah and coauthors in the papers [5], [6] and [11]. We will undertake a different derivation of the effective model, following the expansions proposed in [15]. It gives equation (4), as a rigorous result.

The plan of the paper is as follows. In Section 2 we give the precise setting of the problem and derive its dimensionless form. Then we present the effective problem in its dimensionless form and the main results of the paper. Finally, the effective dispersion problem in its full dimensional form is presented. In Section 3 we recall some facts about the vector-valued Laplace transform and give the Laplace transform of our problem. It permits us to get precise estimates on the transformed solution.

In Subsection 4.1 we present the formal derivation of the hyperbolic effective problem. Even though our approach is different from the one proposed by Balakatoiah et al in [5], [6] and [11], we obtain the same effective dispersion model. In Subsection 4.2 existence, uniqueness and estimates explicit in $\varepsilon$ for the solution to the effective problem are obtained. These estimates are used in Section 5 to prove weak convergence. In Section 6 we add a boundary layer at the inflow boundary. This allows us to prove strong convergence . 
For Péclet numbers close to the threshold value, we are able to obtain a better approximation with hyperbolic effective equation (4) than with the parabolic model (2)- (3). The latter was rigorously justified in [24]. In that paper we needed boundary layer corrections, which complicated the analysis enormously. In the present approach these mathematical technical problems are avoided. Therefore in conclusion: effective equation (4) is a better approximation in the mathematical sense (in the sense of estimates) and easier to justify.

\section{Setting of the problem and main result}

To fix ideas, we give the precise setting of the problem. We consider the transport of a solute by diffusion and convection by Poiseuille's velocity in a semi-infinite two-dimensional channel. The solute particles do not react among themselves nor with the walls. Therefore we suppose zero flux conditions at the lateral walls. The case when the solute undergoes an adsorption process at the lateral boundary will be considered in a forthcoming paper.

We consider the following model for the solute concentration $c^{*}$ :

a) transport through channel $\Omega^{*}=\left\{\left(x^{*}, y^{*}\right): 0<x^{*}<+\infty,\left|y^{*}\right|<H\right\}$

$$
\frac{\partial c^{*}}{\partial t^{*}}+V\left(y^{*}\right) \frac{\partial c^{*}}{\partial x^{*}}-D_{m o l} \frac{\partial^{2} c^{*}}{\partial\left(x^{*}\right)^{2}}-D_{m o l} \frac{\partial^{2} c^{*}}{\partial\left(y^{*}\right)^{2}}=0 \quad \text { in } \Omega^{*},
$$

where $V(z)=Q^{*}\left(1-\left(y^{*} / H\right)^{2}\right)$ and where $Q^{*}$ (velocity) and $D_{m o l}$ (molecular diffusion) are positive constants.

b) zero flux at channel wall $\Gamma^{*}=\left\{\left(x^{*}, y^{*}\right): 0<x^{*}<+\infty,\left|y^{*}\right|=H\right\}$

$$
-D_{m o l} \partial_{y^{*}} c^{*}=0 \quad \text { on } \Gamma^{*},
$$

c) infiltration with a pulse of water containing a solute of concentration $c_{f}^{*}$, followed by solute-free water is stated using the Danckwerts boundary condition from $[16]$

$$
-D_{m o l} \partial_{x^{*}} c^{*}+V\left(y^{*}\right) c^{*}= \begin{cases}V\left(y^{*}\right) c_{f}^{*}, & \text { for } 0<t^{*}<t_{0}^{*} \\ 0, & \text { for } t>t_{0}^{*}\end{cases}
$$

For problems posed on finite interval, we will replace $\Omega^{*}$ by $\Omega_{L}^{*}=\left\{\left(x^{*}, y^{*}\right)\right.$ : $\left.0<x^{*}<L,\left|y^{*}\right|<H\right\}$ and $\Gamma^{*}$ by $\Gamma_{L}^{*}=\left\{\left(x^{*}, y^{*}\right): 0<x^{*}<L,\left|y^{*}\right|=H\right\}$ in (11), (12). In such setting, at $x^{*}=L$ we impose the following boundary condition

$$
-D_{m o l} \partial_{x^{*}} c^{*}=0 \quad \text { on } x^{*}=L, y \in(0, H) .
$$

The natural way of analyzing this problem is to introduce appropriate scales. This requires characteristic or reference values for the parameters in variables involved. The obvious transversal length scale is $H$. For all other quantities we use reference values denoted by the subscript $R$. Setting

$$
c=\frac{c^{*}}{\hat{c}}, x=\frac{x^{*}}{L_{R}}, y=\frac{y^{*}}{H}, t=\frac{t^{*}}{T_{R}}, Q^{*}=Q_{R}, D_{m o l}=D_{R},
$$


where $L_{R}$ is the " observation distance ", we obtain the dimensionless equations

$$
\frac{\partial c}{\partial t}+\frac{Q^{*} T_{R}}{L_{R}}\left(1-y^{2}\right) \frac{\partial c}{\partial x}-\frac{D^{*} T_{R}}{L_{R}^{2}} \frac{\partial^{2} c}{\partial x^{2}}-\frac{D^{*} T_{R}}{H^{2}} \frac{\partial^{2} c}{\partial y^{2}}=0 \quad \text { in } \Omega
$$

and

$$
-\frac{D^{*} T_{R}}{H^{2}} \frac{\partial c}{\partial y}=0 \quad \text { on } \Gamma
$$

where

$$
\Omega=(0,+\infty) \times(-1,1) \text { and } \Gamma=(0,+\infty) \times\{-1,1\} .
$$

The problem involves the following time scales:

$$
\begin{aligned}
& T_{L}=\text { characteristic longitudinal time scale }=\frac{L_{R}}{Q^{*}}, \\
& T_{T}=\text { characteristic transversal time scale }=\frac{H^{2}}{D^{*}},
\end{aligned}
$$

and the dimensionless number $\mathbf{P e}=\frac{L_{R} Q^{*}}{D_{m o l}} \quad$ (Peclet number). In this paper we fix the reference time by setting $T_{R}=T_{L}$. We are going to investigate the behavior of the two-dimensional system (16)-(17) with respect to the small parameter $\varepsilon=\frac{H}{L_{R}}$.

To carry out the analysis we need to compare the dimensionless numbers with respect to $\varepsilon$. For this purpose we set $\mathbf{P e}=\mathbf{P e}_{0} \varepsilon^{-\alpha}$. Introducing the dimensionless numbers in equations (16)-(17) yields the problem:

$$
\begin{gathered}
\mathbf{P e}_{0}\left(\frac{\partial c^{\varepsilon}}{\partial t}+\left(1-y^{2}\right) \frac{\partial c^{\varepsilon}}{\partial x}\right)=\varepsilon^{\alpha} \frac{\partial^{2} c^{\varepsilon}}{\partial x^{2}}+\varepsilon^{\alpha-2} \frac{\partial^{2} c^{\varepsilon}}{\partial y^{2}} \text { in } \Omega^{+} \times(0, T) \\
-\varepsilon^{\alpha-2} \frac{\partial c^{\varepsilon}}{\partial y}=0 \text { on } \Gamma^{+} \times(0, T) \\
c^{\varepsilon}(x, y, 0)=c_{0}(x, y) \text { for }(x, y) \in \Omega^{+} \\
-\frac{\varepsilon^{\alpha}}{\mathbf{P e}_{0}} \partial_{x} c^{\varepsilon}+\left(1-y^{2}\right) c^{\varepsilon}=\left\{\begin{array}{ll}
\left(1-y^{2}\right) c_{f}(t), & \text { for } 0<t<t_{0} \\
0, & \text { for } t>t_{0} .
\end{array} \text { at }\{x=0\}\right. \\
\frac{\partial c^{\varepsilon}}{\partial y}(x, 0, t)=0, \text { for }(x, t) \in(0,+\infty) \times(0, T) .
\end{gathered}
$$

The latter condition results from the $y$-symmetry of the solution. Further

$$
\Omega^{+}=(0,+\infty) \times(0,1), \quad \Gamma^{+}=(0,+\infty) \times\{1\},
$$

and $T$ is an arbitrary chosen positive number.

We study the behavior of this problem as $\varepsilon \searrow 0$, while keeping $\mathbf{P e}_{0}$ of order $\mathcal{O}(1)$. We are only interested in the case $2>\alpha>1$ which leads to dominant hyperbolic behavior. Note that Taylor's data from [29] correspond to $\alpha=1.7$ 
and $\alpha=1.9$. We refer to [15] for detailed discussion about data, expansions and simulations.

Specifically, as in [23], [24], [12] and [13], we will derive expressions for the effective values of the dispersion coefficient and velocity, and an effective one dimensional dispersion equation for small values of $\varepsilon$. The main difference is that here the effective equation will be hyperbolic.

In this paper we suppose $c_{0}=c_{0}(x)$ and prove that the correct upscaling of the Laplace transform of the problem (19)-(23) gives the following effective problem:

$$
\begin{gathered}
\tau c^{0}+<v>\partial_{x} c^{0}-m \varepsilon^{2-\alpha} \mathbf{P} \mathbf{e}_{0} \tau \partial_{x} c^{0}=F \quad \text { in }(0,+\infty), \\
F=\int_{0}^{1} c_{0}(x, y) d y-m \varepsilon^{2-\alpha} \mathbf{P e}_{0} \partial_{x} \int_{0}^{1} c_{0}(x, y) d y . \\
\left.c^{0}\right|_{x=0}=\hat{c}_{f}-\frac{\mathbf{P e}_{0} m \varepsilon^{2-\alpha}\left(\left.c_{0}\right|_{x=0}-\tau \hat{c}_{f}\right)}{<v>-\tau \mathbf{P} \mathbf{e}_{0} m \varepsilon^{2-\alpha}},
\end{gathered}
$$

where $\langle v\rangle=\int_{0}^{1} v(y) d y=\frac{2}{3}, m=\left\langle v(y) P_{4}(y)\right\rangle=-\frac{4}{315}, c_{f}=0$ for $t>t_{0}$ and $P_{4}(y)=\frac{3}{2}\left(\frac{y^{2}}{6}-\frac{y^{4}}{12}-\frac{7}{180}\right)$ is the solution for

$$
\left\{\begin{array}{l}
-\partial_{y y} P_{4}(y)=-\frac{v(y)-<v>}{<v>} \text { on }(0,1), \\
\partial_{y} P_{4}=0 \text { on } y=0 \text { and }-\partial_{y} P_{4}=0 \text { on } y=1 \\
\int_{0}^{1} P_{4}(y) d y=0 .
\end{array}\right.
$$

Remark 1. Derivation of the ODE (24) is given in details in subsection 4.1. Obtaining of the effective boundary conditions at the inlet boundary $x=0$, is independent of the inner expansion developped in 4.1 and leading to the effective PDE.

Let us give a formal derivation of the boundary condition (26): We suppose that $\left.c^{0}\right|_{x=0}=\hat{c}_{f}+\varepsilon^{2-\alpha} c_{f 1}$. then the corresponding correction term at $x=0$ is of the form

$$
\begin{aligned}
& -\frac{v(y)}{\mathbf{P e}_{0}} c_{f 1}-v(y) P_{4}(y)\left(c_{0}(0)-\tau\left(\hat{c}_{f}+\varepsilon^{2-\alpha} c_{f 1}\right)\right)= \\
& -v(y)\left\{\left(P_{4}(y)-\frac{m}{<v>}\right)\left(c_{0}(0)-\tau \hat{c}_{f}-\varepsilon^{2-\alpha} \tau c_{f 1}\right)+\right. \\
& \left.\left(\frac{1}{\mathbf{P e}_{0}}-\frac{m}{<v>} \varepsilon^{2-\alpha} \tau\right) c_{f 1}+\frac{m}{<v>}\left(c_{0}(0)-\tau \hat{c}_{f}\right)\right\} .
\end{aligned}
$$

The above expression has zero mean if and only if $c_{f 1}$ is chosen as

$$
c_{f 1}=-\frac{\mathbf{P e}_{0} m\left(\left.c_{0}\right|_{x=0}-\tau \hat{c}_{f}\right)}{\left\langle v>-\tau \mathbf{P e}_{0} m \varepsilon^{2-\alpha}\right.} .
$$


After inverting the Laplace transform and supposing that $c_{0}(0)=c_{f}(0)$, we get the following one dimensional Goursat's problem for the dimensionless effective concentration $c^{\text {eff }}$ :

$$
\left\{\begin{array}{l}
\partial_{t} c^{e f f}+<v>\partial_{x} c^{e f f}-m \mathbf{P e}_{0} \varepsilon^{2-\alpha} \frac{\partial^{2} c^{e f f}}{\partial x \partial t}=0 \text { for }(x, t) \in \mathbb{R}_{+}^{2}, \\
\left.c^{e f f}\right|_{x=0}=c_{f}(t)-\int_{0}^{t} e^{<v>(t-z)) /\left(\mathbf{P e}_{0} m \varepsilon^{2-\alpha}\right)} \partial_{z} c_{f} d z \\
\partial_{x} c^{e f f} \in L^{2}((0,+\infty) \times(0, T)),\left.\quad c^{e f f}\right|_{t=0}=c_{0}(x)
\end{array}\right.
$$

Let us announce our main result.

Theorem 1. Let $2>\alpha>4 / 3$, let $c_{0} \in H^{2}\left(\mathbb{R}_{+}\right)$and let $c_{f} \in C^{\infty}[0, T]$. Let $c^{\text {eff }}$ be given by (EFF) and let $c^{0}$ be its Laplace transform given by (24)-(26). Then we have

$$
\begin{gathered}
\frac{\varepsilon^{\alpha-2}}{\mathbf{P e}_{0}}\left(\hat{c}^{\varepsilon}-c^{0}\right)-P_{4}(y)\left(<c_{0}>-\tau c^{0}\right) \rightarrow 0 \quad \text { weakly in } L^{2}\left(\Omega^{+}\right), \quad \text { as } \varepsilon \rightarrow 0, \\
\forall \tau \in \mathbb{C}, \Re \tau>0,
\end{gathered}
$$

where $\hat{c}^{\varepsilon}$ is the Laplace transform of $c^{\varepsilon}$.

Let us suppose in addition $c_{f}(0)=c_{0}(0)$. Then the above convergence takes place in $H^{2}\left(\mathbb{C}_{+} ; L^{2}\left(\Omega^{+}\right)\right)$and for all $T \in(0,+\infty)$ we have

$\frac{\varepsilon^{\alpha-2}}{\mathbf{P e}_{0}}\left(c^{\varepsilon}-c^{\text {eff }}\right)+P_{4}(y) \frac{\partial c^{e f f}}{\partial t} \rightarrow 0$ weakly in $L^{2}\left(\Omega^{+} \times(0, T)\right)$, as $\varepsilon \rightarrow 0$.

Our result could be restated in dimensional form:

Corollary 1. Let us suppose that $L_{R}>>\max \left\{D_{m o l} / Q^{*}, Q^{*} H^{2} / D_{m o l}, H\right\}$. Then the upscaled dimensional approximation for (11) reads

$$
\begin{gathered}
\frac{\partial c^{*, e f f}}{\partial t^{*}}+<V>\frac{\partial c^{*, e f f}}{\partial x^{*}}-m H \mathbf{P e}_{T} \frac{\partial^{2} c^{*, e f f}}{\partial x^{*} \partial t^{*}}=0, \text { in } \mathbb{R}_{+} \times(0, T), \\
\left.c^{*, e f f}\right|_{t=0}=c_{0}(x), \\
\left.c^{*, e f f}\right|_{x=0}=c_{f}^{*}\left(t^{*}\right)-\int_{0}^{t^{*}} e^{\left(<V>\left(t^{*}-z^{*}\right)\right) /\left(\mathbf{P e}_{T} m H\right)} \partial_{z^{*}} c_{f}^{*}\left(z^{*}\right) d z^{*},
\end{gathered}
$$

where $\mathbf{P e}_{T}=\frac{Q^{*} H}{D_{m o l}}$ is the transversal Peclet number, $\langle V\rangle=\frac{1}{H} \int_{0}^{H} V(y) d y$ and $H \mathbf{P e}_{T}$ is the mixing length.

Remark 2. At this point it is good to note that equation (30) describes the effective model. However, its solution $c^{* \text {,eff }}$ is only the zeroth order term in asymptotic expansion (46). In order to have the higher order approximation, it is necessary to use

$$
c^{*, \text { approx }}\left(x^{*}, y^{*}, t^{*}\right)=c^{*, e f f}\left(x^{*}, t^{*}\right)-\frac{\partial c^{*, e f f}}{\partial t^{*}}\left(x^{*}, t^{*}\right) \frac{H^{2}}{D_{m o l}} P_{4}\left(\frac{y^{*}}{H}\right)
$$


Finally, with little more work we have the following strong convergence result

Theorem 2. Let $2>\alpha>4 / 3$, let $c_{0} \in H^{2}\left(\mathbb{R}_{+}\right)$and let $c_{f} \in C^{\infty}[0, T]$. Let $c^{e f f}$ be given by (EFF) and let $c^{0}$ be its Laplace transform given by (24)-(26). Then we have

$$
\begin{gathered}
\frac{\varepsilon^{\alpha-2}}{\mathbf{P e}_{0}}\left(\hat{c}^{\varepsilon}-c^{0}\right)-P_{4}(y)\left(<c_{0}>-\tau c^{0}\right) \rightarrow 0 \quad \text { in } \quad L^{2}\left(\Omega^{+}\right), \quad \text { as } \varepsilon \rightarrow 0, \\
\forall \tau \in \mathbb{C}, \Re \tau>0,
\end{gathered}
$$

where $\hat{c}^{\varepsilon}$ is the Laplace transform of $c^{\varepsilon}$.

Let us suppose in addition $c_{f}(0)=c_{0}(0)$. Then the above convergence takes place in $H^{2}\left(\mathbb{C}_{+} ; L^{2}\left(\Omega^{+}\right)\right)$and for all $T \in(0,+\infty)$ we have

$$
\frac{\varepsilon^{\alpha-2}}{\mathbf{P e}_{0}}\left(c^{\varepsilon}-c^{e f f}\right)+P_{4}(y) \frac{\partial c^{e f f}}{\partial t} \rightarrow 0 \quad \text { in } \quad L^{2}\left(\Omega^{+} \times(0, T)\right), \quad \text { as } \varepsilon \rightarrow 0 .
$$

Remark 3. We note that previous results apply to the case of problem (11)-(13) posed for $x \in(0, L)$ and with the outlet boundary condition (14). The statements of the results and the proofs are identical.

\section{Vector valued Laplace transform and applica- tions to PDEs}

We start this section by recalling the basic facts about applications of Laplace's transform to linear parabolic equations. The Laplace's transform method is widely used in solving engineering problems. In applications it is usually called the operational calculus or Heaviside's method.

For locally integrable function $f \in L_{l o c}^{1}(\mathbb{R})$ such that $f(t)=0$ for $t<0$ and $|f(t)| \leq A e^{a t}$ as $t \rightarrow+\infty$, the Laplace transform of $f$, denoted $\hat{f}$, is defined as

$$
\hat{f}(\tau)=\int_{0}^{+\infty} f(t) e^{-\tau t} d t, \quad \tau=\xi+i \eta \in \mathbb{C} .
$$

It is closely linked with Fourier's transform in $\mathbb{R}$. We note that

$$
\hat{f}(\tau)=\mathcal{F}\left(f(t) e^{-\xi t}\right)(-\eta), \quad \xi>a,
$$

where the Fourier's transform of a function $g \in L^{1}(\mathbb{R})$ is given by

$$
\mathcal{F}(g(t))(\omega)=\int_{\mathbb{R}} g(t) e^{i \omega t} d t, \quad \omega \in \mathbb{R} .
$$

It is well-known (see e.g. [31] or [14]) that $\hat{f}$ defined by (36) is analytic in the half-plane $\{\operatorname{Re}(\tau)=\xi>a\}$ and it tends to zero as $\operatorname{Re}(\tau) \rightarrow+\infty$. 
For real applications, Laplace's transform of functions is not well-adapted and it is natural to use Laplace's transform of distributions. It is defined for distributions with support on $[a,+\infty)$ i.e. for $f \in \mathcal{D}_{+}^{\prime}(a)$, where $\mathcal{D}_{+}^{\prime}(a)=\{f \in$ $\left.\mathcal{D}^{\prime}(\mathbb{R}) ; \operatorname{supp} f \subset[a,+\infty)\right\}$. If $\mathcal{S}^{\prime}(\mathbb{R})$ denotes the space of distributions of slow growth, then we introduce $\mathcal{S}_{+}^{\prime}(\mathbb{R})$ by

$$
\mathcal{S}_{+}^{\prime}(\mathbb{R})=\mathcal{D}_{+}^{\prime}(0) \cap \mathcal{S}^{\prime}(\mathbb{R})
$$

and we use the formula (37) to define Laplace's transform for $f \in \mathcal{D}_{+}^{\prime}(a)$ such that $f e^{-\xi t} \in \mathcal{S}_{+}^{\prime}(\mathbb{R})$ for all $\xi>a$. This approach permits the rigorous operational calculus. For details we refer to classical textbooks as [31] by Vladimirov.

Laplace's transform is applier to linear ODEs and PDEs, the transform problem is solved and its solution $\hat{f}$ is calculated. Then the important question is how to inverse the Laplace's transform. First we need a suitable space for image functions. It is the algebra $H(a)$ defined by

$$
\begin{aligned}
H(a)=\{\quad & g \in \mathcal{H o l}(\{\tau \in \mathbb{C} ; \operatorname{Re}(\tau)>a\}) \text { satisfying the growth condition : } \\
& \text { for any } \sigma_{o}>a \text { there are real numbers } C\left(\sigma_{o}\right)>0 \text { and } m=m\left(\sigma_{o}\right) \geq 0 \\
& \text { such that } \left.|g(\tau)| \leq C\left(\sigma_{o}\right)\left(1+|\tau|^{m}\right), \operatorname{Re}(\tau)>\sigma_{o}\right\} .
\end{aligned}
$$

For elements of $H(a)$ we have the following classical result.

Theorem 3. ([31] pp. 162-165) Let $\hat{f} \in H(a)$ be absolutely integrable with respect to $\eta$ on $\mathbb{R}$ for certain $\xi>a$. Then the following formula holds true.

$$
f(t)=\frac{1}{2 \pi i} \int_{\xi-i \infty}^{\xi+i \infty} \hat{f}(z) e^{z t} d z .
$$

These classical results are not sufficient for our purposes. We need results for reflexive Sobolev space $X$ valued Laplace's transform. Furthermore we need an inversion theorem in $L^{p}((0,+\infty) ; X)$. The corresponding theory could be found in Arendt [2] and we give only results directly linked to our needs. For a reflexive Banach space $X$ we set

$\mathcal{C}_{w}^{\infty}\left(\mathbb{R}_{+} ; X\right)=\left\{r \in \mathcal{C}^{\infty}((0,+\infty) ; X) ;\|r\|_{w}=\operatorname{supsup}_{n \in \mathbb{N} \lambda>0} \frac{\lambda^{n+1}}{n !}\left\|\frac{d^{n}}{d \lambda^{n}} r(\lambda)\right\|_{X}<+\infty\right\}$.

Then we have the following result.

Theorem 4. ([2], Chapter 2) Let $X$ be a reflexive Banach space. Then the (real) Laplace's transform $f \mapsto \hat{f}$ is an isometric isomorphism between $L^{\infty}\left(\mathbb{R}_{+} ; X\right)$ and $\mathcal{C}_{w}^{\infty}\left(\mathbb{R}_{+} ; X\right)$.

Let $X$ be a Hilbert space, $\mathbb{C}_{+}=\{\lambda \in \mathbb{C}: \operatorname{Re} \lambda>0\}$ and let $H^{2}\left(\mathbb{C}_{+}, X\right)$ be the subset of the space of holomorphic functions defined by

$H^{2}\left(\mathbb{C}_{+}, X\right)=\left\{h: \mathbb{C}_{+} \rightarrow X\right.$ such that $\left.\|h\|_{H^{2}\left(\mathbb{C}_{+}, X\right)}=\sup _{x>0} \int_{\mathbb{R}}\|h(x+i s)\|_{X}^{2} d s<+\infty\right\}$.

Then we have 
Theorem 5. (vector valued Paley-Wiener theorem from [2], page 48) Let $X$ be a Hilbert space. Then the map $\left.f \rightarrow \hat{f}\right|_{\mathbb{C}}$ is an isometric isomorphism of $L^{2}\left(\mathbb{R}_{+}, X\right)$ onto $H^{2}\left(\mathbb{C}_{+}, X\right)$.

In our situation, we have to deal with $\mathbb{C}_{+}$replaced by $\{\lambda \in \mathbb{C}: \operatorname{Re} \lambda>$ $\left.\tau_{0}>0\right\}$. But this means just replacing $f$ by $e^{-\tau t} f$ in Theorem 5 . Other, more direct way to proceed is to follow ideas from [14] and use a direct approach based on the link to Fourier's transform. We apply this result in the study of the upscaled equations and then in the error estimates. We derive estimates for the solutions of the Laplace transformed problem.

\section{Rigorous derivation and analysis of the effec- tive problem}

\subsection{Formal asymptotic expansion}

We suppose $\alpha \geq 1$.

Let the operator $\mathcal{L}^{\varepsilon}$ be given by

$$
\mathcal{L}^{\varepsilon} \zeta=\tau \zeta+\left(1-y^{2}\right) \frac{\partial \zeta}{\partial x}-\frac{\varepsilon^{\alpha}}{\mathbf{P e}_{0}}\left(\frac{\partial^{2} \zeta}{\partial x^{2}}+\varepsilon^{-2} \frac{\partial^{2} \zeta}{\partial y^{2}}\right) .
$$

The dimensionless physical concentration $c^{\varepsilon}$ satisfies (19)-(23). Its Laplace transform $\hat{c}^{\varepsilon}$ is thus solution of

$$
\begin{gathered}
\mathcal{L}^{\varepsilon} \hat{c}^{\varepsilon}=c_{0} \quad \text { in }(0,+\infty) \times(0,1) \\
-\frac{\varepsilon^{\alpha}}{\mathbf{P e}_{0}} \partial_{x} \hat{c}^{\varepsilon}+\left(1-y^{2}\right) \hat{c}^{\varepsilon}=\left(1-y^{2}\right) \hat{c}_{f}, \quad \text { for }(x, y) \in\{0\} \times(0,1), \\
-\frac{\varepsilon^{\alpha-2}}{\mathbf{P e}_{0}} \partial_{y} \hat{c}^{\varepsilon}(x, y, \tau)=0 \quad \text { on }(0,+\infty) \times(\{0\} \cup\{1\}) .
\end{gathered}
$$

We start from the system (43)-(45) and search for $\hat{c}^{\varepsilon}$ in the form

$$
\hat{c}^{\varepsilon}=c^{0}(x, t ; \varepsilon)+\varepsilon^{2-\alpha} c^{1}(x, y, t)+\varepsilon^{2(2-\alpha)} c^{2}(x, y, t)+\ldots
$$

After introducing (46) into the equation (43) we get

$$
\begin{gathered}
\varepsilon^{0}\left\{\tau c^{0}+\left(1-y^{2}\right) \partial_{x} c^{0}-\frac{1}{\mathbf{P e}_{0}} \partial_{y y} c^{1}-c_{0}\right\}+\varepsilon^{2-\alpha}\left\{\tau c^{1}+\right. \\
\left.\left(1-y^{2}\right) \partial_{x} c^{1}--\frac{1}{\mathbf{P e}_{0}} \partial_{y y} c^{2}\right\}=O\left(\varepsilon^{2(2-\alpha)}\right)+O\left(\varepsilon^{\alpha}\right) .
\end{gathered}
$$

In order to have (47) for every $\varepsilon \in\left(0, \varepsilon_{0}\right)$, all coefficients in front of the powers of $\varepsilon$ should be zero. 
The problem corresponding to the order $\varepsilon^{0}$ is

$$
\left\{\begin{array}{l}
-\frac{1}{\mathbf{P e}_{0}} \partial_{y y} c^{1}=-\left(1 / 3-y^{2}\right) \partial_{x} c^{0}+c_{0}-\int_{0}^{1} c_{0}(x, y) d y \\
-\left(\tau c^{0}+2 \partial_{x} c^{0} / 3-\int_{0}^{1} c_{0}(x, y) d y\right) \text { on }(0,1) \\
\partial_{y} c^{1}=0 \text { on } y=0 \text { and }-\partial_{y} c^{1}=0 \text { on } y=1
\end{array}\right.
$$

for every $x \in(0,+\infty)$. By Fredholm's alternative, the problem (48) has a solution if and only if

$$
\tau c^{0}+2 \partial_{x} c^{0} / 3-\int_{0}^{1} c_{0}(x, y) d y=0 \quad \text { in }(0,+\infty)
$$

For $\alpha$ close to 2, the equation (49) gives a coarse approximation and it does not suit our needs. It is interesting to include some higher order terms and get better approximation. We proceed as in [23] and [15], following an idea from [27], and suppose that

$$
\tau c^{0}+2 \partial_{x} c^{0} / 3-\int_{0}^{1} c_{0}(x, y) d y=O\left(\varepsilon^{2-\alpha}\right) \quad \text { in }(0,+\infty) .
$$

The hypothesis (50) will be justified a posteriori, after getting an equation for $c^{0}$. It is convenient to use (50) and write the right hand side of the first equation in $(48)$ as

$$
\begin{gathered}
-\left(1 / 3-y^{2}\right) \partial_{x} c^{0}-\left(\tau c^{0}+2 \partial_{x} c^{0} / 3-\int_{0}^{1} c_{0}(x, y) d y\right)= \\
-\frac{3}{2}\left(\frac{1}{3}-y^{2}\right)\left(\int_{0}^{1} c_{0}(x, y) d y-\tau c^{0}\right)+O\left(\varepsilon^{2-\alpha}\right) \quad \text { in }(0,+\infty) .
\end{gathered}
$$

Let $\pi(x, y), \int_{0}^{1} \pi(x, y) d y=0$, be the unique solution to the problem

$$
-\partial_{y y} \pi=c_{0}(x, y)-\int_{0}^{1} c_{0}(x, y) d y \quad \text { on }(0,1),\left.\quad \partial_{y} \pi\right|_{y=0,1}=0 .
$$

Then (48) reduces to

$$
\left\{\begin{array}{l}
-\frac{1}{\mathbf{P e}_{0}} \partial_{y y} c^{1}=-\frac{3}{2}\left(1 / 3-y^{2}\right)\left(\int_{0}^{1} c_{0}(x, y) d y-\tau c^{0}\right) \\
+c_{0}(x, y)-\int_{0}^{1} c_{0}(x, y) d y \text { on }(0,1) \\
\partial_{y} c^{1}=0 \text { on } y=0 \text { and }-\partial_{y} c^{1}=0 \text { on } y=1
\end{array}\right.
$$

for every $x \in(0,+\infty)$, and we have

$$
\frac{1}{\mathbf{P e}_{0}} c^{1}(x, y, t)=\frac{3}{2}\left(\frac{y^{2}}{6}-\frac{y^{4}}{12}-\frac{7}{180}\right)\left(\int_{0}^{1} c_{0}(x, y) d y-\tau c^{0}\right)+\pi(x, y)+C_{0}(x, t),
$$


where $C_{0}$ is an arbitrary function.

Let us go to the next order. Then we have

$$
\left\{\begin{array}{l}
-\frac{1}{\mathbf{P e}_{0}} \partial_{y y} c^{2}=-\left(1-y^{2}\right) \partial_{x} c^{1}-\tau c^{1}-\varepsilon^{\alpha-2}\left(\tau c^{0}+2 \partial_{x} c^{0} / 3-\right. \\
\left.\int_{0}^{1} c_{0}(x, y) d y\right) \quad \text { on }(0,1), \\
\partial_{y} c^{2}=0 \text { on } y=0 \text { and }-\partial_{y} c^{2}=0 \text { on } y=1
\end{array}\right.
$$

for every $x \in(0,+\infty)$. The problem (55) has a solution if and only if

$$
\begin{gathered}
\tau c^{0}+2 \partial_{x} c^{0} / 3-\int_{0}^{1} c_{0}(x, y) d y+\varepsilon^{2-\alpha} \partial_{x}\left(\int_{0}^{1}\left(1-y^{2}\right) c^{1} d y\right)+ \\
\varepsilon^{2-\alpha} \tau\left(\int_{0}^{1} c^{1} d y\right)-\frac{\varepsilon^{2}}{\mathbf{P e}_{0}} \partial_{x x}\left(\int_{0}^{1} c^{1} d y\right)=0 \quad \text { in }(0,+\infty) .
\end{gathered}
$$

(56) is the equation for $c^{0}$. Obviously, $c^{0}+\frac{\varepsilon^{2-\alpha}}{\mathbf{P e}_{0}} C_{0}$ satisfies the same equation at order $O\left(\varepsilon^{2(2-\alpha)}\right)$ and we choose $C_{0}=0$ without loosing generality.

Finally, after straightforward calculations, the equation (56) becomes (24)(25), with slightly more general $F$ given by

$$
F=\int_{0}^{1} c_{0}(x, y) d y+\varepsilon^{2-\alpha} \mathbf{P e}_{0} \partial_{x}\left(\frac{4}{315} \int_{0}^{1} c_{0}(x, y) d y-\int_{0}^{1}\left(1-y^{2}\right) \pi(x, y) d y\right) .
$$

\subsection{Study of the upscaled diffusion-convection equation on the half-line}

In Section 5, we will prove that the original problem can be approximated by an upscaled one dimensional diffusion-convection equation. The present section is thus devoted to the study of this type of equation in the half-line. The results of Subsection 4.2 are used in Section 5.

For $\bar{Q}, \bar{D}$ and $\gamma>0$, we consider the problem

$$
\left\{\begin{array}{l}
\partial_{t} u+\bar{Q} \partial_{x} u+\gamma \bar{D} \partial_{x t} u=G \text { in }(0,+\infty) \times(0, T) \\
\partial_{x} u \in L^{2}((0,+\infty) \times(0, T)), \\
u(x, 0)=u_{0} \text { in }(0,+\infty), \quad u=c_{f} \text { at } x=0
\end{array}\right.
$$

Let $\Omega_{l}=\mathbb{R}_{+} \times\{\operatorname{Re}(\tau)>0\}$. After applying the Laplace transform with respect to the time variable we get the following equation for the Laplace transform $\hat{u}(x, \tau)$ of $u$ :

$$
\left\{\begin{array}{l}
\tau \hat{u}+\bar{Q} \partial_{x} \hat{u}+\gamma \bar{D} \tau \partial_{x} \hat{u}=\bar{F}+\gamma U_{0}=\hat{G}+u_{0}(x)+\gamma \bar{D} \partial_{x} u_{0} \text { in } \Omega_{l} \\
\partial_{x} \hat{u} \in L^{2}\left(\mathbb{R}_{+}\right), \quad \operatorname{Re}(\tau)>0 \\
\hat{u}=\hat{c}_{f} \text { at } x=0
\end{array}\right.
$$


where $\tau=\xi+i \eta \in \mathbb{C}, \xi>0$. In order to capture correctly the decay in $\tau$, we transform the problem (59) into the following problem for the unknown $\hat{v}=\hat{u}-\frac{u_{0}}{\beta+\tau}, \beta>0$,

$\left\{\begin{array}{l}\tau \hat{v}+\bar{Q} \partial_{x} \hat{v}+\gamma \bar{D} \tau \partial_{x} \hat{v}=\hat{G}+\frac{\beta u_{0}-\bar{Q} \partial_{x} u_{0}}{\beta+\tau}+\gamma \bar{D} \frac{\beta \partial_{x} u_{0}}{\beta+\tau} \text { in } \Omega_{l}, \\ \partial_{x} \hat{v} \in L^{2}\left(\mathbb{R}_{+}\right), \quad \operatorname{Re}(\tau)>0, \\ \hat{u}=\hat{c}_{f}-\frac{u_{0}}{\beta+\tau} \text { at } x=0 .\end{array}\right.$

We decompose $\hat{v}$ as $\hat{v}=\hat{a}+\gamma \bar{D} \frac{\beta}{\beta+\tau} \hat{f}$, with

$$
\left\{\begin{array}{l}
\tau \hat{a}+\bar{Q} \partial_{x} \hat{a}+\gamma \bar{D} \tau \partial_{x} \hat{a}=\hat{G}+\frac{\beta u_{0}-\bar{Q} \partial_{x} u_{0}}{\beta+\tau} \text { in } \Omega_{l} \\
\partial_{x} \hat{a} \in L^{2}\left(\mathbb{R}_{+}\right), \quad \operatorname{Re}(\tau)>0 \\
\hat{a}=\hat{c}_{f}-\frac{u_{0}}{\beta+\tau} \text { at } x=0
\end{array}\right.
$$

and

$$
\left\{\begin{array}{l}
\tau \hat{f}+\bar{Q} \partial_{x} \hat{f}+\gamma \bar{D} \tau \partial_{x} \hat{f}=\partial_{x} u_{0} \text { in } \Omega_{l}, \\
\partial_{x} \hat{f} \in L^{2}\left(\mathbb{R}_{+}\right), \quad \operatorname{Re}(\tau)>0 \\
\hat{f}=0 \text { at } x=0 .
\end{array}\right.
$$

For the sake of simplicity, we write

$$
\ell(\tau)=\bar{Q}+\gamma \tau \bar{D}, \quad \operatorname{Re}(\tau)=\xi>0
$$

We have for $\xi>0$

$$
\begin{gathered}
\operatorname{Re}\left(\frac{\tau}{l(\tau)}\right)=\operatorname{Re}\left(\frac{\tau \overline{l(\tau)}}{|l(\tau)|^{2}}\right)=\frac{\xi \bar{Q}+\gamma \bar{D}|\tau|^{2}}{(\gamma \bar{D})^{2}|\tau|^{2}+\bar{Q}^{2}+2 \gamma \bar{D} \bar{Q} \xi}>0 \\
1 / \operatorname{Re}\left(\frac{\tau}{l(\tau)}\right)=\gamma \bar{D}+\bar{Q} \frac{\bar{Q}+\gamma \bar{D} \xi}{\gamma \bar{D}|\tau|^{2}+\bar{Q} \xi} \\
\frac{1}{|\ell(\tau)|}=\frac{1}{\sqrt{\bar{Q}^{2}+(\gamma \bar{D})^{2}|\tau|^{2}}} \leq \frac{\sqrt{2}}{\bar{Q}+\gamma \bar{D}|\tau|}
\end{gathered}
$$


Problem (61) for $\hat{a}$ allows the following explicit solution:

$$
\begin{gathered}
\hat{a}(x, \tau)=\left(\hat{c}_{f}(\tau)-\left.\frac{u_{0}}{\beta+\tau}\right|_{x=0}\right) e^{-\tau x / \ell(\tau)}+\int_{0}^{x} e^{-\tau(x-z) / \ell(\tau)} \frac{\hat{G}(z, \tau)}{\ell(\tau)} d z \\
+\int_{0}^{x} \frac{\beta u_{0}(x-z)-\bar{Q} \partial_{x} u_{0}(x-z)}{(\beta+\tau) \ell(\tau)} e^{-\tau z / \ell(\tau)} d z \\
\partial_{x} \hat{a}(x, \tau)=\frac{1}{\ell(\tau)}\left(\left.u_{0}\right|_{x=0}-\tau \hat{c}_{f}(\tau)-\frac{\left.\left.\bar{Q} \partial_{x} u_{0}\right|_{x=0}\right) e^{-\tau x / \ell(\tau)}+}{\beta+\tau}\right. \\
\int_{0}^{x} \frac{\beta \partial_{x} u_{0}(x-z)-\bar{Q} \partial_{x x} u_{0}(x-z)}{(\beta+\tau) \ell(\tau)} e^{-\tau z / \ell(\tau)} d z-\int_{0}^{x} e^{-\tau(x-z) / \ell(\tau)} \frac{\tau \hat{G}(z, \tau)}{\ell^{2}(\tau)} d z
\end{gathered}
$$

Problem (62) for $\hat{f}$ allows the following explicit solution:

$$
\begin{gathered}
\hat{f}(x, \tau)=\int_{0}^{x} e^{-\tau(x-z) / \ell(\tau)} \frac{\partial_{x} u_{0}(z, \tau)}{\ell(\tau)} d z \\
\partial_{x} \hat{f}(x, \tau)=-\frac{\tau \partial_{x} u_{0}(x)}{\ell^{2}(\tau)}-\int_{0}^{x} \frac{\tau \partial_{x} u_{0}(z)}{\ell^{2}(\tau)} e^{-\tau(x-z) / \ell(\tau)} d z .
\end{gathered}
$$

This explicit formula allows us to find the exact behavior of $u$ with respect to $\gamma$.

We now aim to give explicit estimates with respect to $\tau$ for $\hat{u}$ in $H^{p}((0,+\infty))$. First, for any $p \in[1,+\infty]$, Young's inequality implies that

$$
\|\hat{u}(\cdot, \tau)\|_{L^{p}\left(\mathbb{R}_{+}\right)} \leq C\left(\left|\hat{c}_{f}\right|+\frac{\left\|u_{0}\right\|_{H^{1}\left(\mathbb{R}_{+}\right)}}{|\tau|}+\|\hat{G}(\cdot, \tau)\|_{L^{2}(0,+\infty)}\right)
$$

Estimating $\partial_{x} \hat{u}$ is more delicate. We start by estimating the terms in the equalities (67) and (69) concentrated at the boundary. Using (63)-(65) we get the following estimate:

$$
\begin{gathered}
\left\|\frac{1}{\ell(\tau)}\left(\left.u_{0}\right|_{x=0}-\tau \hat{c}_{f}(\tau)-\frac{\left.\bar{Q} \partial_{x} u_{0}\right|_{x=0}}{\beta+\tau}\right) e^{-\tau x / \ell(\tau)}-\gamma \bar{D} \frac{\beta}{\beta+\tau} \frac{\tau \partial_{x} u_{0}(x)}{\ell^{2}(\tau)}\right\|_{L^{p}\left(\mathbb{R}_{+}\right)} \leq \\
C\left\{\left|u_{0}\right|_{x=0}-c_{f}(0)|+| \tau \hat{c}_{f}-c_{f}(0) \mid+\frac{\left|\partial_{x} u_{0}\right|_{x=0} \mid}{|\tau|}+\frac{\left\|\partial_{x} u_{0}\right\|_{L^{2}\left(\mathbb{R}_{+}\right)}}{|\tau|}\right\} .
\end{gathered}
$$

Next we estimate the convolution terms using Young's inequality and (63)-(65):

$$
\begin{gathered}
\| \int_{0}^{x} \frac{\beta \partial_{x} u_{0}(x-z)-\bar{Q} \partial_{x x} u_{0}(x-z)}{(\beta+\tau) \ell(\tau)} e^{-\tau z / \ell(\tau)} d z-\int_{0}^{x} e^{-\tau(x-z) / \ell(\tau)} \frac{\tau \hat{G}(z, \tau)}{\ell^{2}(\tau)} \\
d z \|_{L^{p}\left(\mathbb{R}_{+}\right)} \leq C\left\{\|\tau \hat{G}(\cdot, \tau)\|_{L^{2}\left(\mathbb{R}_{+}\right)}+\frac{\left\|\partial_{x} u_{0}\right\|_{H^{1}\left(\mathbb{R}_{+}\right)}}{|\tau|}\right\} \\
\left\|\gamma \bar{D} \frac{\beta}{\beta+\tau} \int_{0}^{x} \frac{\tau \partial_{x} u_{0}(z)}{\ell^{2}(\tau)} e^{-\tau(x-z) / \ell(\tau)} d z\right\|_{L^{p}\left(\mathbb{R}_{+}\right)} \leq C \frac{\left\|\partial_{x} u_{0}\right\|_{L^{2}\left(\mathbb{R}_{+}\right)}}{|\tau|}
\end{gathered}
$$

The following estimates are then straightforward: 
Lemma 1. Let

$$
u_{0} \in H^{2}\left(\mathbb{R}_{+}\right), c_{f} \in H^{1}\left(\mathbb{R}_{+}\right) \quad \text { and } G \in H_{0}^{1}\left(\mathbb{R}_{+}\right) .
$$

Then the problem (59) has a unique solution $\hat{u} \in L^{2}\left(\mathbb{R}_{+} ; H^{1}\left(\mathbb{R}_{+}\right)\right)$satisfying (70) and

$$
\begin{gathered}
\left\|\partial_{x} \hat{u}(\cdot, \tau)\right\|_{L^{p}((0,+\infty))} \leq C\left\{\left|u_{0}\right|_{x=0}-c_{f}(0)|+| \tau \hat{c}_{f}-c_{f}(0) \mid+\frac{\left\|\partial_{x} u_{0}\right\|_{L^{2}\left(\mathbb{R}_{+}\right)}}{|\tau|}\right. \\
\left.+\|\tau \hat{G}(\cdot, \tau)\|_{L^{2}\left(\mathbb{R}_{+}\right)}+\frac{\left\|\partial_{x} u_{0}\right\|_{H^{1}\left(\mathbb{R}_{+}\right)}}{|\tau|}\right\}
\end{gathered}
$$

with $\tau=\xi+i \eta, \xi>0$ and $p \in[1,+\infty]$. Constant $C$ does not depend on $\gamma$.

Let in addition to (74) the data satisfy the compatibility condition $u_{0}(0)=$ $c_{f}(0)$. Then the problem (58) has a unique solution $u \in H^{1}\left(\mathbb{R}_{+}^{2}\right)$ satisfying

$$
\begin{gathered}
\left\|\partial_{x} u\right\|_{L^{2}\left(\mathbb{R}_{+}^{2}\right)}=\left\|\partial_{x} \hat{u}\right\|_{H^{2}\left(\mathbb{C}_{+}, L^{2}((0,+\infty))\right.} \leq C\left\{\left\|c_{f}\right\|_{H^{1}\left(\mathbb{R}_{+}\right)}+\|G\|_{H^{1}\left(\mathbb{R}_{+}\right)}+\right. \\
\left.\left\|u_{0}\right\|_{H^{2}\left(\mathbb{R}_{+}\right)}\right\} .
\end{gathered}
$$

Next we estimate the the expression $\tau \hat{u}-u_{0}$. We have $\tau \hat{u}-u_{0}=\tau \hat{v}-\frac{\beta u_{0}}{\tau+\beta}$. As before $\hat{v}$ is decomposed as $\hat{v}=\hat{a}+\frac{\gamma \bar{D} \beta}{\tau+\beta} \hat{f}$, where $\hat{a}$ is given by (61) and $\hat{f}$ by (62). Direct calculation gives

$$
\begin{aligned}
& \tau \hat{a}(x, \tau)=\left(\hat{c}_{f}(\tau)-\left.u_{0}\right|_{x=0}+\left.\frac{\bar{Q} \partial_{x} u_{0}}{\beta+\tau}\right|_{x=0}\right) e^{-\tau x / \ell(\tau)}+\int_{0}^{x} e^{-\tau(x-z) / \ell(\tau)} \frac{\tau \hat{G}(z, \tau)}{\ell(\tau)} d z \\
& +\int_{0}^{x} \frac{\beta \partial_{x} u_{0}(x-z)-\bar{Q} \partial_{x x} u_{0}(x-z)}{(\beta+\tau)} e^{-\tau z / \ell(\tau)} d z+\frac{\beta u_{0}(x)-\bar{Q} \partial_{x} u_{0}(x)}{\tau+\beta} ; \\
& \tau \partial_{x} \hat{a}(x, \tau)=\frac{\tau}{\ell(\tau)}\left(\left.u_{0}\right|_{x=0}-\tau \hat{c}_{f}(\tau)-\frac{\left.\bar{Q} \partial_{x} u_{0}\right|_{x=0}}{\beta+\tau}\right) e^{-\tau x / \ell(\tau)}+\frac{\tau \hat{G}(x, \tau)}{\ell(\tau)}- \\
& \int_{0}^{x} \frac{\beta \partial_{x} u_{0}(x-z)-\bar{Q} \partial_{x x} u_{0}(x-z)}{\beta+\tau} e^{-\tau z / \ell(\tau)} d z-\int_{0}^{x} e^{-\tau(x-z) / \ell(\tau)} \frac{\tau^{2} \hat{G}(z, \tau)}{\ell^{2}(\tau)} d z \\
& +\frac{\beta \partial_{x} u_{0}(x)-\bar{Q} \partial_{x x} u_{0}(x)}{\tau+\beta}+\frac{\left.\beta \partial_{x} u_{0}\right|_{x=0}-\left.\bar{Q} \partial_{x x} u_{0}\right|_{x=0}}{\tau+\beta} e^{-\tau x / \ell(\tau)} .
\end{aligned}
$$

For the second component $\hat{f}$ we have

$$
\begin{gathered}
\tau \hat{f}(x, \tau)=-\int_{0}^{x} e^{-\tau z / \ell(\tau)} \partial_{x x} u_{0}(x-z, \tau) d z+\partial_{x} u_{0}(x) \\
\tau \partial_{x} \hat{f}(x, \tau)=-\left.\int_{0}^{x} e^{-\tau x / \ell(\tau)} \partial_{x} u_{0}\right|_{x=0} ; \\
\left.+\left.\frac{\tau}{\ell(\tau)} e^{-\tau x / \ell(\tau)} \partial_{x x x} u_{0} u_{0}\right|_{x=0} . z, \tau\right) d z+\partial_{x x} u_{0}(x)
\end{gathered}
$$

Equalities (77) and (79) imply the following result 
Lemma 2. Let us suppose the assumption (74)on the data. Then, as in Lemma 1 the problem (59) has a unique solution $\hat{u} \in L^{2}\left(\mathbb{R}_{+} ; H^{1}\left(\mathbb{R}_{+}\right)\right)$satisfying (70), (75) and

$$
\begin{gathered}
\left\|u_{0}-\tau \hat{u}\right\|_{L^{p}((0,+\infty))} \leq C\left\{\left|u_{0}\right|_{x=0}-c_{f}(0)|+| \tau \hat{c}_{f}-c_{f}(0) \mid+\frac{\left\|\partial_{x} u_{0}\right\|_{H^{1}\left(\mathbb{R}_{+}\right)}}{|\tau|}\right. \\
\left.+\|\tau \hat{G}(\cdot, \tau)\|_{L^{2}\left(\mathbb{R}_{+}\right)}+\frac{\left\|\partial_{x} u_{0}\right\|_{H^{1}\left(\mathbb{R}_{+}\right)}}{|\tau|}\right\}
\end{gathered}
$$

with $\tau=\xi+i \eta, \xi>0$ and any $p \in[1,+\infty]$. Constant $C$ does not depend on $\gamma$

Let in addition to (74) the data satisfy the compatibility condition $u_{0}(0)=$ $c_{f}(0)$. Then the problem (58) has a unique solution $u \in H^{1}\left(\mathbb{R}_{+}^{2}\right)$ satisfying (76) and

$$
\begin{gathered}
\left\|\partial_{t} u\right\|_{L^{2}\left(\mathbb{R}_{+}^{2}\right)}=\left\|u_{0}-\tau \hat{u}\right\|_{H^{2}\left(\mathbb{C}_{+}, L^{2}((0,+\infty))\right.} \leq C\left\{\left\|c_{f}\right\|_{H^{1}\left(\mathbb{R}_{+}\right)}+\|G\|_{H^{1}\left(\mathbb{R}_{+}\right)}+\right. \\
\left.\left\|u_{0}\right\|_{H^{2}\left(\mathbb{R}_{+}\right)}\right\} .
\end{gathered}
$$

It remains to study the limit $\gamma \rightarrow 0$. Let $u_{\gamma}=u$ be given by (58). Let $u_{\gamma 0}$ satisfies (58) with $\gamma=0$. Then we have

$$
\begin{gathered}
\hat{u}_{\gamma}(x, \tau)=\hat{u}(x, \tau) \rightarrow \hat{u}_{\gamma 0}(x, \tau), \quad \forall x \in \mathbb{R}_{+} \text {and } \forall \tau \in \mathbb{C}_{+}, \\
u_{0}-\tau \hat{u}_{\gamma}(x, \tau)=u_{0}-\tau \hat{u}(x, \tau) \rightarrow u_{0}-\tau \hat{u}_{\gamma 0}(x, \tau), \quad \forall x \in \mathbb{R}_{+} \text {and } \forall \tau \in \mathbb{C}_{+}, \\
\partial_{x} \hat{u}_{\gamma}(x, \tau)=\partial_{x} \hat{u}(x, \tau) \rightarrow \partial_{x} \hat{u}_{\gamma 0}(x, \tau), \quad \forall x \in \mathbb{R}_{+} \text {and } \forall \tau \in \mathbb{C}_{+} .
\end{gathered}
$$

Next, under the hypothesis (74) and with the compatibility condition $u_{0}(0)=$ $c_{f}(0)$, Lemmas 1 and 2 are valid and sequences $\left|\hat{u}_{\gamma}\right|^{2},\left|u_{0}-\tau \hat{u}_{\gamma}\right|^{2}$ and $\left|\partial_{x} \hat{u}_{\gamma}\right|^{2}$ are bounded from above by a non-negative function integrable with respect to $x$ and $\eta=\operatorname{Im} \tau$ and bounded with respect to $\xi=\Re \tau$. Consequently, Lebesgue's dominated convergence theorem gives

Lemma 3. Let us suppose the assumption (74)on the data and let, furthermore, the data satisfy the compatibility condition $u_{0}(0)=c_{f}(0)$. Let $u_{\gamma}=u \in H^{1}\left(\mathbb{R}_{+}^{2}\right)$ be the unique solution for the problem (58), satisfying estimates (76) and (82). Let $u_{\gamma 0} \in H^{1}\left(\mathbb{R}_{+}^{2}\right)$ be the unique solution for the problem (58) with $\gamma=0$. Then we have

$$
\begin{gathered}
\hat{u}_{\gamma} \rightarrow \hat{u}_{\gamma 0}, \quad u_{0}-\tau \hat{u}_{\gamma} \rightarrow u_{0}-\tau \hat{u}_{\gamma 0} \quad \text { and } \quad \partial_{x} \hat{u}_{\gamma} \rightarrow \partial_{x} \hat{u}_{\gamma 0} \\
\text { in } H^{2}\left(\mathbb{C}_{+}, L^{2}((0,+\infty)) \text {, as } \gamma \rightarrow 0,\right. \\
u_{\gamma} \rightarrow u_{\gamma 0}, \partial_{t} u_{\gamma} \rightarrow \partial_{t} u_{\gamma 0} \text { and } \partial_{x} u_{\gamma} \rightarrow \partial_{x} u_{\gamma 0} \text { in } L^{2}\left(\mathbb{R}_{+}^{2}\right) \text {, as } \gamma \rightarrow 0 .
\end{gathered}
$$

\section{Proof of Theorem 1}

\section{STEP 1}


Let $H^{1}\left(\Omega^{+}\right)$be the usual Sobolev space, but complex valued. Let $v(y)=$ $1-y^{2}$ and $c_{0}(x, y)=c_{0}(x)=<c_{0}>$. We write the problem (43)-(45) in the variational form

$$
\begin{gathered}
\int_{\Omega^{+}} \tau \hat{c}^{\varepsilon} \varphi d x d y+\int_{\Omega^{+}} v(y) \partial_{x} \hat{c}^{\varepsilon} \varphi d x d y+\frac{\varepsilon^{\alpha}}{\mathbf{P} \mathbf{e}_{0}} \int_{\Omega^{+}}\left(\partial_{x} \hat{c}^{\varepsilon} \partial_{x} \varphi+\varepsilon^{-2} \partial_{y} \hat{c}^{\varepsilon} \partial_{y} \varphi\right) d x d y \\
+\left.\left.\int_{0}^{1} v(y) \hat{c}^{\varepsilon}\right|_{x=0} \varphi\right|_{x=0} d y=\int_{\Omega^{+}} c_{0} \varphi d x d y+\left.\int_{0}^{1} v(y) \hat{c}_{f} \varphi\right|_{x=0} d y \\
\forall \varphi \in H^{1}\left(\Omega^{+}\right), \quad \forall \tau \in \mathbb{C}_{+}
\end{gathered}
$$

Next let $P_{4}(y)=\frac{3}{2}\left(\frac{y^{2}}{6}-\frac{y^{4}}{12}-\frac{7}{180}\right)$ and $m=\int_{0}^{1} v(y) P_{4}(y) d y=\left\langle v P_{4}\right\rangle=$ $-4 / 315$. We write the problem (24)-(26) in the variational form

$$
\begin{gathered}
\int_{\Omega^{+}} \tau c^{0} \varphi d x d y+\int_{\Omega^{+}} v(y) \partial_{x} c^{0} \varphi d x d y+\frac{\varepsilon^{\alpha}}{\mathbf{P e}_{0}} \int_{\Omega^{+}}\left(\partial_{x} c^{0} \partial_{x} \varphi+\varepsilon^{-2} \partial_{y} c^{0} \partial_{y} \varphi\right) d x d y \\
+\left.\left.\int_{0}^{1} v(y) c^{0}\right|_{x=0} \varphi\right|_{x=0} d y=\int_{\Omega^{+}} c_{0} \varphi d x d y+\left.\int_{0}^{1} v(y) \hat{c}_{f} \varphi\right|_{x=0} d y- \\
\int_{\Omega^{+}} m \varepsilon^{2-\alpha} \mathbf{P e}_{0} \partial_{x}<c_{0}>\varphi d x d y+\frac{\varepsilon^{\alpha}}{\mathbf{P e}_{0}} \int_{\Omega^{+}} \partial_{x} c^{0} \partial_{x} \varphi d x d y+ \\
\quad \int_{\Omega^{+}}(v(y)-<v>) \partial_{x} c^{0} \varphi d x d y+\int_{\Omega^{+}} \varepsilon^{2-\alpha} \mathbf{P e}_{0} m \tau \partial_{x} c^{0} \varphi d x d y- \\
\left.\int_{0}^{1} v(y) \frac{\mathbf{P e}_{0} m \varepsilon^{2-\alpha}\left(\left.c_{0}\right|_{x=0}-\tau \hat{c}_{f}\right)}{<v>-\mathbf{P e}_{0} m \tau \varepsilon^{2-\alpha}} \varphi\right|_{x=0} d y, \quad \forall \varphi \in H^{1}\left(\Omega^{+}\right), \quad \forall \tau \in \mathbb{C}_{+} . \quad \text { (89) }
\end{gathered}
$$

It should be noticed that the equation (24) is the first order partial differential equation in $x$ and the $3 \mathrm{rd}$ and the 4th term at the left hand side of (89) are added to allow the comparaison with (88). They do not come from integration by parts and the test functions do not have to satisfy the boundary condition at $x=0$.

Then the function $q^{\varepsilon}=\varepsilon^{\alpha-2}\left(\hat{c}^{\varepsilon}-c^{0}\right) / \mathbf{P e}_{0}$ satisfies the following variational equation

$$
\begin{gathered}
b\left(q^{\varepsilon}, \bar{\varphi}\right)=\int_{\Omega^{+}} \tau q^{\varepsilon} \varphi d x d y+\left.\left.\int_{0}^{1} v(y) q^{\varepsilon}\right|_{x=0} \varphi\right|_{x=0} d y+\int_{\Omega^{+}} v(y) \partial_{x} q^{\varepsilon} \varphi d x d y+ \\
\frac{\varepsilon^{\alpha}}{\mathbf{P e}_{0}} \int_{\Omega^{+}}\left(\partial_{x} q^{\varepsilon} \partial_{x} \varphi+\varepsilon^{-2} \partial_{y} q^{\varepsilon} \partial_{y} \varphi\right) d x d y=\varepsilon^{\alpha-2} \int_{\Omega^{+}} \frac{<v>-v(y)}{\mathbf{P e}_{0}} \partial_{x} c^{0} \varphi d x d y \\
-\int_{\Omega^{+}} m \tau \partial_{x} c^{0} \varphi d x d y+\int_{\Omega^{+}} m \partial_{x} c_{0} \varphi d x d y-\frac{\varepsilon^{2(\alpha-1)}}{\mathbf{P e}_{0}^{2}} \int_{\Omega^{+}} \partial_{x} c^{0} \partial_{x} \varphi d x d y+ \\
\left.\int_{0}^{1} v(y) \frac{m\left(\left.c_{0}\right|_{x=0}-\tau \hat{c}_{f}\right)}{<v>-\mathbf{P e}_{0} m \tau \varepsilon^{2-\alpha}} \varphi\right|_{x=0} d y, \forall \varphi \in H^{1}\left(\Omega^{+}\right), \forall \tau \in \mathbb{C}_{+}
\end{gathered}
$$

\section{STEP 2}


We use $\varphi=\bar{q}^{\varepsilon}$ (the complex conjugate of $q^{\varepsilon}$ ) as test function in (90). Then using the results from Sections 4.1-4.2, we have

$$
\begin{aligned}
& \left|\varepsilon^{\alpha-2} \int_{\Omega^{+}} \frac{v(y)-<v>}{\mathbf{P e}_{0}} \partial_{x} c^{0} \bar{q}^{\varepsilon} d x d y\right| \leq\left|\varepsilon^{\alpha / 2-1} \int_{\Omega^{+}} \frac{\partial_{y} P_{4}}{\mathbf{P e}_{0}} \partial_{x} c^{0} \varepsilon^{\alpha / 2-1} \partial_{y} \bar{q}^{\varepsilon} d x d y\right| \\
& \leq C \varepsilon^{\alpha / 2-1}\left\|\partial_{x} c^{0}\right\|_{L^{2}\left(\Omega^{+}\right)}\left\|\varepsilon^{\alpha / 2-1} \partial_{y} \bar{q}^{\varepsilon}\right\|_{L^{2}\left(\Omega^{+}\right)} \\
& \left|\int_{\Omega^{+}} \mathbf{P e}_{0} m \tau \partial_{x} c^{0} \bar{q}^{\varepsilon} d x d y\right| \leq C|\tau||| \partial_{x} c^{0}\left\|_{L^{2}\left(\Omega^{+}\right)}\right\| \bar{q}^{\varepsilon} \|_{L^{2}\left(\Omega^{+}\right)} \\
& \left|\int_{\Omega^{+}} \mathbf{P e}_{0} \partial_{x} m<c_{0}>+\bar{q}^{\varepsilon} d x d y\right| \leq C\left\|c_{0}\right\|_{H^{1}\left(\Omega^{+}\right)}|| \bar{q}^{\varepsilon} \|_{L^{2}\left(\Omega^{+}\right)} \\
& \frac{\varepsilon^{2(\alpha-1)}}{\mathbf{P e}_{0}^{2}}\left|\int_{\Omega^{+}} \partial_{x} c^{0} \partial_{x} \bar{q}^{\varepsilon} d x d y\right| \leq C \varepsilon^{3 \alpha / 2-2}\left\|\partial_{x} c^{0}\right\|_{L^{2}\left(\Omega^{+}\right)}|| \varepsilon^{\alpha / 2} \partial_{x} \bar{q}^{\varepsilon} \|_{L^{2}\left(\Omega^{+}\right)}, \\
& \left|\int_{0}^{1} \frac{\left.m v(y)\left(\left.c_{0}\right|_{x=0}-\tau \hat{c}_{f}\right) \varphi\right|_{x=0}}{<v>-\mathbf{P e}_{0} m \tau \varepsilon^{2-\alpha}} d y\right| \leq C\left|c_{0}\right|_{x=0}-\tau \hat{c}_{f}\left|\left\|\left.\sqrt{v(y)} \varphi\right|_{x=0}\right\|_{L^{2}(0,1)}\right.
\end{aligned}
$$

Estimates (91)-(95) give a precise behavior of the right hand side in (90). We note that for $\alpha \geq 1$ one has $3 \alpha / 2-2 \geq \alpha / 2-1$, and from (91)-(95)we get

$$
\begin{gathered}
\left\|\partial_{y} q^{\varepsilon}\right\|_{L^{2}\left(\Omega^{+}\right)} \leq C\left\{\left\|c_{0}\right\|_{H^{1}\left(\Omega^{+}\right)}+\left|c_{0}\right|_{x=0}-\tau \hat{c}_{f} \mid+\right. \\
\left.\left(1+|\tau|^{1 / 2} \varepsilon^{1-\alpha / 2}\right)\left\|\partial_{x} c^{0}\right\|_{L^{2}\left(\Omega^{+}\right)}\right\}
\end{gathered}
$$

Estimate (96) implies existence of a subsequence of $\left\{q^{\varepsilon}\right\}$, denoted by the same superscript, and $Q \in L^{2}\left(\Omega^{+}\right)$, such that $\partial_{y} q^{\varepsilon} \rightarrow Q$ weakly in $L^{2}\left(\Omega^{+}\right)$. Passing to the limit in the variational equation (90), yields the following equation for $Q$ :

$$
\int_{\Omega^{+}} Q \partial_{y} \varphi d x d y=-\int_{\Omega^{+}}(v(y)-<v>) \partial_{x} c^{0, t r} \varphi d x d y
$$

where $c^{0, t r}$ is the solution for the problem

$$
\left\{\begin{array}{l}
\tau c^{0, t r}+<v>\partial_{x} c^{0, t r}=<c_{0}>\quad \text { in } \Omega^{+} \\
\left.c^{0, t r}\right|_{x=0}=\hat{c}_{f} .
\end{array}\right.
$$

Next we obtain that

$$
\partial_{y} q^{\varepsilon}=\varepsilon^{\alpha-2} \partial_{y} \frac{\hat{c}^{\varepsilon}-c^{0}}{\mathbf{P e}_{0}} \rightarrow \partial_{y} P_{4}(y)\left(<c_{0}>-\tau c^{0, t r}\right),
$$

weakly in $L^{2}\left(\Omega^{+}\right)$, for every $\tau \in \mathbb{C}_{+}$. This is in accordance with the results by Choquet and Mikelić from [12]. 
With $c_{0}(0)=c_{f}(0)$ we use Lemma 1 and conclude that

$$
\begin{gathered}
\varepsilon^{\alpha-2} \partial_{y} \frac{\hat{c}^{\varepsilon}-c^{0}}{\mathbf{P e}_{0}} \rightarrow \partial_{y} P_{4}(y)\left(<c_{0}>-\tau c^{0, t r}\right) \text { in } H^{2}\left(\mathbb{C}_{+}, L^{2}(0,+\infty)\right), \\
\varepsilon^{\alpha-2} \partial_{y} \frac{c^{\varepsilon}-c^{e f f}}{\mathbf{P e}_{0}} \longrightarrow-\partial_{y} P_{4}(y) \partial_{t} c^{t r} \text { in } L^{2}\left(\mathbb{R}_{+}^{2}\right) .
\end{gathered}
$$

We would like to go one step forward, extend the results from [12] and use the hyperbolic effective equation to prove the weak convergence of $q^{\varepsilon}$ to $P_{4}(y)\left(<c_{0}>-\tau c^{0, t r}\right)$.

\section{STEP 3}

Having in mind the estimates from STEP 2, we introduce the function $w^{\varepsilon}$ by

$$
w^{\varepsilon}=\frac{\varepsilon^{\alpha-2}}{\mathbf{P e}_{0}}\left(\hat{c}^{\varepsilon}-c^{0}\right)-P_{4}(y)\left(<c_{0}>-\tau c^{0}\right)
$$

and write the corresponding variational equation.

After inserting (102) into (90) and using the identity

$$
\int_{\Omega^{+}} \frac{v(y)-<v>}{\mathbf{P e}_{0}} \partial_{x} c^{0} \varphi d x d y+\int_{\Omega^{+}} \frac{\partial_{y} P_{4}}{\mathbf{P e}_{0}}<v>\partial_{x} c^{0} \partial_{y} \varphi d x d y=0
$$

we get the following variational equation for $w^{\varepsilon}$ :

$$
\begin{gathered}
\frac{1}{\tau} b\left(w^{\varepsilon}, \bar{\varphi}\right)=-\int_{\Omega^{+}} P_{4}(y)\left(<c_{0}>-\tau c^{0}\right) \varphi d x d y- \\
\int_{\Omega^{+}}\left(v(y) P_{4}(y)-m\right) \partial_{x}\left(\frac{<c_{0}>}{\tau}-c^{0}\right) \varphi d x d y-\frac{\varepsilon^{2(\alpha-1)}}{\mathbf{P e}_{0}} \int_{\Omega^{+}} \partial_{x} \frac{c^{0}}{\tau} \partial_{x} \varphi d x d y \\
-\frac{\varepsilon^{\alpha}}{\mathbf{P e}_{0}} \int_{\Omega^{+}} P_{4}(y) \partial_{x}\left(\frac{<c_{0}>}{\tau}-c^{0}\right) \partial_{x} \varphi d x d y-\int_{0}^{1} v(y)\left(P_{4}(y)-\right. \\
\left.\frac{m}{<v>}\right)\left.\left(\frac{<c_{0}>\left.\right|_{x=0}}{\tau}-\left.c^{0}\right|_{x=0}\right) \varphi\right|_{x=0} d y+m \int_{\Omega^{+}} \partial_{x}\left(c^{0}-\right. \\
\left.\frac{<c_{0}>}{\tau}\right) \partial_{y} P_{4} \partial_{y} \varphi d x d y, \quad \forall \varphi \in H^{1}\left(\Omega^{+}\right), \quad \forall \tau \in \mathbb{C}_{+} .
\end{gathered}
$$


Let us estimate the terms at the right hand side:

$$
\begin{aligned}
& \left|\int_{\Omega^{+}} P_{4}(y)\left(<c_{0}>-\tau c^{0}\right) \varphi d x d y\right| \leq C||<c_{0}>-\tau c^{0}\left\|_{L^{2}\left(\Omega^{+}\right)}\right\| \varphi \|_{L^{2}\left(\Omega^{+}\right)}, \\
& \left|\int_{\Omega^{+}}\left(v(y) P_{4}(y)-m\right) \partial_{x}\left(\frac{<c_{0}>}{\tau}-c^{0}\right) \varphi d x d y\right| \leq \\
& \frac{C \varepsilon^{1-\alpha / 2}}{\sqrt{\Re\left(\frac{1}{\tau}\right)}}\left\|\partial_{x}\left(\frac{<c_{0}>}{\tau}-c^{0}\right)\right\|_{L^{2}\left(\Omega^{+}\right)}\left\|\sqrt{\Re\left(\frac{1}{\tau}\right)} \varepsilon^{\alpha / 2-1} \partial_{y} \varphi\right\|_{L^{2}\left(\Omega^{+}\right)}, \\
& \left|\frac{\varepsilon^{2(\alpha-1)}}{\mathbf{P e}_{0}} \int_{\Omega^{+}} \partial_{x} \frac{c^{0}}{\tau} \partial_{x} \varphi d x d y\right| \leq \\
& \frac{C}{|\tau|} \frac{\varepsilon^{3 \alpha / 2-2}}{\sqrt{\Re\left(\frac{1}{\tau}\right)}}\left\|\partial_{x} c^{0}\right\|_{L^{2}\left(\Omega^{+}\right)}\left\|\sqrt{\Re\left(\frac{1}{\tau}\right)} \varepsilon^{\alpha / 2} \partial_{x} \varphi\right\|_{L^{2}\left(\Omega^{+}\right)}, \\
& \frac{\varepsilon^{\alpha}}{\mathbf{P e}_{0}}\left|\int_{\Omega^{+}} P_{4}(y) \partial_{x}\left(\frac{<c_{0}>}{\tau}-c^{0}\right) \partial_{x} \varphi d x d y\right| \leq \\
& \frac{C \varepsilon^{\alpha / 2}}{\sqrt{\Re\left(\frac{1}{\tau}\right)}}\left\|\partial_{x}\left(\frac{<c_{0}>}{\tau}-c^{0}\right)\right\|_{L^{2}\left(\Omega^{+}\right)}\left\|\sqrt{\Re\left(\frac{1}{\tau}\right)} \varepsilon^{\alpha / 2} \partial_{x} \varphi\right\|_{L^{2}\left(\Omega^{+}\right)}, \\
& \left|\int_{0}^{1} v(y)\left(P_{4}(y)-\frac{m}{<v>}\right)\left(\frac{<c_{0}>\left.\right|_{x=0}}{\tau}-\left.c^{0}\right|_{x=0}\right) \varphi\right|_{x=0} d y \mid \leq \\
& \frac{C}{|\tau| \sqrt{\Re\left(\frac{1}{\tau}\right)}}\left|<c_{0}>\right| x_{x=0}-\left.\tau \hat{c}_{f}\left|\| \sqrt{\Re\left(\frac{1}{\tau}\right)} \sqrt{v(y)} \varphi\right|_{x=0}\right|_{L^{2}(0,1)}, \\
& \left.\mid m \int_{\Omega^{+}} \partial_{x}\left(-c^{0}+\frac{<c_{0}>}{\tau}\right)\right) \partial_{y} P_{4} \partial_{y} \varphi d x d y \mid \leq \\
& \frac{C \varepsilon^{1-\alpha / 2}}{\sqrt{\Re\left(\frac{1}{\tau}\right)}}\left\|\partial_{x}\left(\frac{<c_{0}>}{\tau}-c^{0}\right)\right\|_{L^{2}\left(\Omega^{+}\right)}\left\|\sqrt{\Re\left(\frac{1}{\tau}\right)} \varepsilon^{\alpha / 2-1} \partial_{y} \varphi\right\|_{L^{2}\left(\Omega^{+}\right)}
\end{aligned}
$$

Therefore, since

$$
\frac{1}{|\tau| \sqrt{\Re\left(\frac{1}{\tau}\right)}} \leq \frac{1}{\sqrt{\Re \tau}}
$$


for $\alpha>4 / 3$, we have

$$
\begin{gathered}
\Re\left(\frac{1}{\tau} b\left(w^{\varepsilon}, w^{\varepsilon}\right)\right)=\int_{\Omega^{+}}\left|w^{\varepsilon}\right|^{2} d x d y+\varepsilon^{\alpha-2} \Re\left(\frac{1}{\tau}\right) \int_{\Omega^{+}}\left|\partial_{y} w^{\varepsilon}\right|^{2} d x d y+ \\
\varepsilon^{\alpha} \Re\left(\frac{1}{\tau}\right) \int_{\Omega^{+}}\left|\partial_{x} w^{\varepsilon}\right|^{2} d x d y+\left.\frac{1}{2} \Re\left(\frac{1}{\tau}\right) \int_{0}^{1}\left|\sqrt{v(y)} w^{\varepsilon}\right|_{x=0}\right|^{2} d y \leq \\
C\left\{\left\|<c_{0}>-\tau c^{0}\right\|_{L^{2}\left(\Omega^{+}\right)}+\varepsilon^{1-\alpha / 2}\left\|\partial_{x}\left(<c_{0}>-\tau c^{0}\right)\right\|_{L^{2}\left(\Omega^{+}\right)}+\right. \\
\left.\varepsilon^{\alpha / 2}\left\|\partial_{x} c^{0}\right\|_{L^{2}\left(\Omega^{+}\right)}+\left|<c_{0}>\right|{ }_{x=0}-\tau \hat{c}_{f} \mid\right\}^{2}
\end{gathered}
$$

The estimate (110) gives

$$
\left\{\begin{array}{l}
\left\|\partial_{y} w^{\varepsilon}\right\|_{L^{2}\left(\Omega^{+}\right)} \leq C \varepsilon^{1-\alpha / 2} ; \quad\left\|w^{\varepsilon}\right\|_{L^{2}\left(\Omega^{+}\right)} \leq C ; \\
\left\|\partial_{x} w^{\varepsilon}\right\|_{L^{2}\left(\Omega^{+}\right)} \leq C \varepsilon^{-\alpha / 2} ; \quad\left\|\left.\sqrt{v} w^{\varepsilon}\right|_{x=0}\right\|_{L^{2}(0,1)} \leq C .
\end{array}\right.
$$

Consequently, there is $w=w(x, \tau) \in L^{2}\left(\mathbb{R}_{+}\right)$, for every $\tau \in \mathbb{C}_{+}$such that

$$
w^{\varepsilon} \rightarrow w \text { in } \quad L^{2}\left(\Omega^{+}\right) \text {and } \partial_{y} w^{\varepsilon} \rightarrow 0 \quad \text { in } \quad L^{2}\left(\Omega^{+}\right), \quad \forall \tau \in \mathbb{C}_{+} .
$$

Next we take the test function depending only on $x, \varphi=\varphi(x) \in H^{1}\left(\mathbb{R}_{+}\right)$. Then we pass to the limit in the variation equation (103). Since $\varphi$ does not depend on $y$ and terms are either small or involve the section mean equal to zero, we obtain that the right hand side converges to zero when $\varepsilon \rightarrow 0$. Then passing to the limit $\varepsilon \rightarrow 0$ in the left side of (103) yields

$$
\int_{0}^{+\infty} \tau w \varphi(x) d x-\int_{0}^{+\infty}<v>w \partial_{x} \varphi(x) d x=0, \quad \forall \varphi \in H^{1}\left(\mathbb{R}_{+}\right) .
$$

The equation (113) yields $w \in H^{1}\left(\mathbb{R}_{+}\right)$and $w(0, \tau)=0$. Therefore $w=0$ on $\mathbb{R}_{+}$for all $\tau \in \mathbb{C}_{+}$. This proves $(28)$.

It remains to prove the convergence with respect to the time as well. Difficulty is that bounds in (111) depend on $\tau$ and their integrability is to be discussed.

We use the results of the Section 4.2. Using estimate (110), assumptions on the data and Lemmas 1 and 2, we obtain that

$$
\left\|w^{\varepsilon}\right\|_{H^{2}\left(\mathbb{C}_{+} ; L^{2}\left(\Omega^{+}\right)\right)}+\varepsilon^{\alpha / 2-1}\left\|\sqrt{\Re\left(\frac{1}{\tau}\right)} \partial_{y} w^{\varepsilon}\right\|_{H^{2}\left(\mathbb{C}_{+} ; L^{2}\left(\Omega^{+}\right)\right)} \leq C .
$$

Now we repeat the above convergence argument but in $H^{2}\left(\mathbb{C}_{+} ; L^{2}\left(\Omega^{+}\right)\right)$and obtain (29). This proves the theorem.

\section{Proof of Theorem 2}

\section{STEP 1}


We construct the corresponding boundary layer through the problem

$$
\begin{gathered}
v(y) \partial_{z} \beta=\partial_{y y} \beta \quad \text { in } \Omega^{+}, \\
\beta(0, y)=P_{4}(y)-\frac{m}{<v>} \text { on }(0,1), \\
\left.\partial_{y} \beta\right|_{y=0,1}=0 \text { for } x \in \mathbb{R}_{+} .
\end{gathered}
$$

Since $\int_{0}^{1} v(y) \beta(0, y) d y=0$, we can apply the elementary separation of variables for the heat equation and conclude $\beta$ has an exponential decay in $z$, i.e. that there is $\lambda_{0}>0$ such that

$$
|\beta(z, y)| \leq C e^{-\lambda_{0} z}, \quad \forall(z, y) \in \Omega^{+} .
$$

For more details on the spectral properties of the problem (116)-(117) we refer to [14], vol. 5, page 63 .

\section{STEP 2}

Next we set

$$
\beta^{\varepsilon}(x, y)=\beta\left(\frac{x}{\varepsilon^{2-\alpha} \mathbf{P e}_{0}}, y\right)
$$

and

$$
w^{\varepsilon, f u l l}=\frac{\varepsilon^{\alpha-2}}{\mathbf{P e}_{0}}\left(\hat{c}^{\varepsilon}-c^{0}\right)-P_{4}(y)\left(<c_{0}>-\tau c^{0}\right)+\beta^{\varepsilon}(x, y)\left(<c_{0}>\left|{ }_{x=0}-\tau c^{0}\right|_{x=0}\right) .
$$

We make the corresponding replacement in (103) and get

$$
\begin{gathered}
\frac{1}{\tau} b\left(w^{\varepsilon, f u l l}, \bar{\varphi}\right)=-\int_{\Omega^{+}} P_{4}(y)\left(<c_{0}>-\tau c^{0}\right) \varphi d x d y+\int_{\Omega^{+}} \beta^{\varepsilon}\left(<c_{0}>\left.\right|_{x=0}-\right. \\
\left.\left.\tau c^{0}\right|_{x=0}\right) \varphi d x d y+\frac{\varepsilon^{\alpha}}{\mathbf{P e}_{0}} \int_{\Omega^{+}}\left(<c_{0}>||_{x=0}-\left.\tau c^{0}\right|_{x=0}\right) \partial_{x} \beta^{\varepsilon} \partial_{x} \varphi d x d y- \\
\int_{\Omega^{+}}\left(v(y) P_{4}(y)-m\right) \partial_{x}\left(\frac{<c_{0}>}{\tau}-c^{0}\right) \varphi d x d y-\frac{\varepsilon^{2(\alpha-1)}}{\mathbf{P e}_{0}} \int_{\Omega^{+}} \partial_{x} \frac{c^{0}}{\tau} \partial_{x} \varphi d x d y \\
-\frac{\varepsilon^{\alpha}}{\mathbf{P e}_{0}} \int_{\Omega^{+}} P_{4}(y) \partial_{x}\left(\frac{<c_{0}>}{\tau}-c^{0}\right) \partial_{x} \varphi d x d y-\int_{0}^{1} v(y)\left(P_{4}(y)-\right. \\
\left.\frac{m}{<v>}\right)\left.\left(\frac{<c_{0}>\mid{ }_{x=0}}{\tau}-\left.c^{0}\right|_{x=0}\right) \varphi\right|_{x=0} d y+m \int_{\Omega^{+}} \partial_{x}\left(c^{0}-\right. \\
\left.\frac{<c_{0}>}{\tau}\right) \partial_{y} P_{4} \partial_{y} \varphi d x d y, \quad \forall \varphi \in H^{1}\left(\Omega^{+}\right), \quad \forall \tau \in \mathbb{C}_{+} .
\end{gathered}
$$

Next we note the modifications in the estimate (104):

$$
\begin{gathered}
\left|\int_{\Omega^{+}} P_{4}(y)\left(<c_{0}>-\tau c^{0}\right) \varphi d x d y\right| \leq \\
\frac{C \varepsilon^{1-\alpha / 2}}{\sqrt{\Re\left(\frac{1}{\tau}\right)}}\left\|<c_{0}>-\tau c^{0}\right\|_{L^{2}\left(\Omega^{+}\right)}\left\|\sqrt{\Re\left(\frac{1}{\tau}\right)} \varepsilon^{\alpha / 2-1} \partial_{y} \varphi\right\|_{L^{2}\left(\Omega^{+}\right)},
\end{gathered}
$$


Furthermore we have two new terms:

$$
\begin{gathered}
\left|\int_{\Omega^{+}} \beta^{\varepsilon}\left(<c_{0}>\left|{ }_{x=0}-\tau c^{0}\right|_{x=0}\right) \varphi d x d y\right| \leq C \\
\varepsilon^{(2-\alpha) / 2}\left|<c_{0}>\right|_{x=0}-\left.\left.\tau c^{0}\right|_{x=0}|||\varphi|\right|_{L^{2}\left(\Omega^{+}\right)} \\
\left|\int_{\Omega^{+}} \frac{\varepsilon^{\alpha}}{\mathbf{P e}_{0}}\left(<c_{0}>\left.\right|_{x=0}-\left.\tau c^{0}\right|_{x=0}\right) \partial_{x} \beta^{\varepsilon} \partial_{x} \varphi d x d y\right| \leq \\
C \varepsilon^{\alpha-1}\left|<c_{0}>\right|_{x=0}-\left.\tau c^{0}\right|_{x=0}|| \mid \varepsilon^{\alpha / 2} \partial_{x} \varphi \|_{L^{2}\left(\Omega^{+}\right)}
\end{gathered}
$$

Now for $\alpha>4 / 3$, we get again

$$
\left\|w^{\varepsilon}\right\|_{L^{2}\left(\Omega^{+}\right)} \leq C e^{\min \{3 \alpha / 2-2,1-\alpha / 2\}} \text { and } w^{\varepsilon} \rightarrow 0 \text { in } L^{2}\left(\Omega^{+}\right), \forall \tau \in \mathbb{C}_{+}
$$

and the (34) is proved.

It remains to prove (35). The only term which needs attention is

$$
\int_{\Omega^{+}} P_{4}(y)\left(<c_{0}>-\tau c^{0}\right) \varphi d x d y .
$$

Here we use Lemma 3 giving us $<c_{0}>-\tau c^{0} \rightarrow<c_{0}>-\tau c^{0, t r}$ in $H^{2}\left(\mathbb{C}_{+} ; L^{2}\left(\Omega^{+}\right)\right)$, as $\varepsilon \rightarrow 0$. Now we take as test function $\varphi=\bar{w}^{\varepsilon, f u l l}$. The test function converges weakly to zero in $H^{2}\left(\mathbb{C}_{+} ; L^{2}\left(\Omega^{+}\right)\right)$, as $\varepsilon \rightarrow 0$. Therefore we have $\Re\left(\frac{1}{\tau} b\left(w^{\varepsilon, \text { full }}, w^{\varepsilon, \text { full }}\right)\right) \rightarrow 0$, as $\varepsilon \rightarrow 0$. This proves (35).

\section{References}

[1] G. Allaire and A.-L. Raphael, Homogenization of a convection - diffusion model with reaction in a porous medium, C. R. Math. Acad. Sci. Paris, Vol. 344,8 (2007), pp. 523-528.

[2] W. Arendt, C.J.K. Batty, M. Hieber and F. Neubrander, Vector-valued Laplace transforms and Cauchy problems, Birkhäuser, Basel, 2001.

[3] R. Aris, On the dispersion of a solute in a fluid flowing through a tube, Proc. Roy. Soc. London Sect A., 235 (1956), pp. 67-77.

[4] V. Balakotaiah and H.-C. Chang, Dispersion of Chemical Solutes in Chromatographs and Reactors, Phil. Trans. R. Soc. Lond. A, Vol. 351, 1695 (1995), pp. 39-75.

[5] V. Balakotaiah, H.-C. Chang, Hyperbolic Homogenized Models for Thermal and Solutal Dispersion, SIAM J. Appl. Maths. , Vol. 63 (2003), p. 12311258 .

[6] V. Balakotaiah, Hyperbolic averaged models for describing dispersion effects in chromatographs and reactors, Korean J. Chem. Eng., Vol. 21, 2 (2004), pp. 318-328. 
[7] C. W. J. Berentsen, M. L. Verlaan, C. P. J. W. van Kruijsdijk, Upscaling and reversibility of Taylor dispersion in heterogeneous porous media, Phys. Rev. E, 71 (2005), pp. 1-16.

[8] J. Camacho, Thermodynamics of Taylor Dispersion: Constitutive equations, Physical Review E, Vol. 47 (1993), nr. 2, p. 1049-1053.

[9] J. Camacho, Purely Global Model for Taylor Dispersion, Physical Review E, Vol. 48 (1993), nr. 1, p. $310-321$.

[10] J. Camacho, Thermodynamics functions for Taylor's dispersion, Physical Review E, Vol. 48 (1993), nr. 3, p. 1844-1849.

[11] S. Chakraborty and V. Balakotaiah, Spatially averaged multi-scale models for chemical reactions, Advances in Chemical Engineering, Vol 30 (2005), pp. 205-297.

[12] C. Choquet, A. Mikelić, Laplace transform approach to the rigorous upscaling of the infinite adsorption rate reactive flow under dominant Peclet number through a pore, Applicable Analysis, Vol. 87, No. 12, December 2008, 1373-1395.

[13] C. Choquet, A. Mikelić, Rigorous upscaling of the reactive flow with finite kinetics and under dominant Peclet number, Continuum Mechanics and Thermodynamics, Volume 21, 2009, p. 125-140.

[14] R. Dautray and J.L. Lions, Analyse mathématique et calcul numérique pour les sciences et techniques, Vol. 5, Spectre des opérateurs; Vol. \%, Evolution : Fourier, Laplace, Masson, Cea, Paris, 1984.

[15] C.J. van Duijn, A. Mikelić, I. S. Pop, C. Rosier, Effective Dispersion Equations For Reactive Flows With Dominant Peclet and Damkohler Numbers, Advances in Chemical Engineering, Vol 34 (2008), pp. 1-45.

[16] M. Th. van Genuchten and R.W. Cleary, Movement of solutes in soil: computer-simulated and laboratory results, chapter 10 in "Soil Chemistry B. Physico-Chemical Models", ed. by G. H. Bolt, Developements in Soil Sciences 5B, Elsevier Scientific Publishing Company, Amsterdam, 1979, pp. 349-386.

[17] G. Karniadakis, A. Beskok, N. Aluru, Microflows and Nanoflows, Springer, New York, 2005.

[18] A.D. Khon'kin, the Taylor and hyperbolic models of unsteady longitudinal dispersion of a passive impurity in convection-diffusion processes, J. Appl. Maths Mechs, Vol. 64, no. 4 (2000), p. 607-617.

[19] B. E. Launder, Turbulent Transport Models for Numerical Computation of Fluid Flow, Dept. Mech. Eng., Univ. of California, Davis (1978). 
[20] C. Maas, A hyperbolic dispersion equation to model the bounds of a contaminated groundwater body, Journal of Hydrology, Vol. 226 (1999), p. 234 $-241$.

[21] R. Mauri, Dispersion, convection and reaction in porous media, Phys. Fluids A (1991), pp. 743-755.

[22] G.N. Mercer and A.J. Roberts, A centre manifold description of contaminant dispersion in channels with varying flow profiles, SIAM J. Appl. Math., Vol. 50 (1990), pp. 1547-1565.

[23] A. Mikelić, V. Devigne and C.J. van Duijn, Rigorous upscaling of the reactive flow through a pore, under dominant Peclet and Damkohler numbers, SIAM J. Math. Anal., Vol. 38, 4 (2006), pp. 1262-1287.

[24] A. Mikelić and C. Rosier: Rigorous upscaling of the infinite adsorption rate reactive flow under dominant Peclet number through a pore, Ann Univ Ferrara Sez. VII Sci. Mat.,Vol. 53 (2007), pp. 333-359.

[25] M.A. Paine, R.G. Carbonell and S. Whitaker, Dispersion in pulsed systems - I, Heterogeneous reaction and reversible adsorption in capillary tubes, Chemical Engineering Science, Vol. 38 (1983), pp. 1781-1793.

[26] S. Rosencrans, Taylor dispersion in curved channels, SIAM J. Appl. Math., Vol. 57 (1997), pp. 1216 - 1241.

[27] J. Rubinstein and R. Mauri, Dispersion and convection in porous media, SIAM J. Appl. Math., Vol. 46 (1986), pp. 1018 - 1023.

[28] A.E. Scheidegger, Typical solutions of the differential equations of statistical theories of flow through porous media. Trans. Am. Geophys. Union, Vol. 39 (1958), p. 929-932.

[29] G.I. Taylor, Dispersion of soluble matter in solvent flowing slowly through a tube, Proc. Royal Soc. A, Vol. 219 (1953), pp. 186-203.

[30] G.I. Taylor, The dispersion of matter in turbulent flow through a pipe, Proc. Royal Soc. A, Vol. 223 (1954), pp. 446-468.

[31] V.S. Vladimirov, Equations of mathematical physics, URSS, Moscow, 1996. 